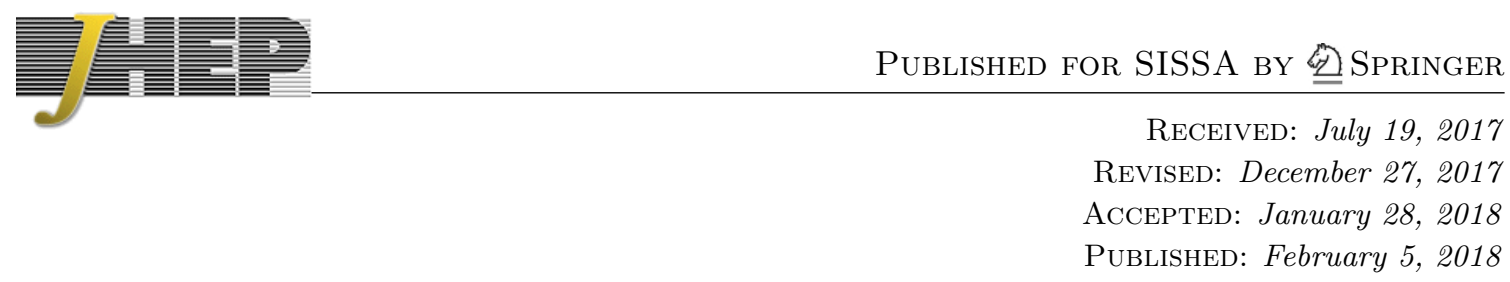

\title{
Local phase space and edge modes for diffeomorphism-invariant theories
}

\author{
Antony J. Speranza \\ Maryland Center for Fundamental Physics, University of Maryland, \\ College Park, MD 20742, U.S.A. \\ E-mail: asperanz@gmail.com
}

ABSTRACT: We discuss an approach to characterizing local degrees of freedom of a subregion in diffeomorphism-invariant theories using the extended phase space of Donnelly and Freidel [36]. Such a characterization is important for defining local observables and entanglement entropy in gravitational theories. Traditional phase space constructions for subregions are not invariant with respect to diffeomorphisms that act at the boundary. The extended phase space remedies this problem by introducing edge mode fields at the boundary whose transformations under diffeomorphisms render the extended symplectic structure fully gauge invariant. In this work, we present a general construction for the edge mode symplectic structure. We show that the new fields satisfy a surface symmetry algebra generated by the Noether charges associated with the edge mode fields. For surface-preserving symmetries, the algebra is universal for all diffeomorphism-invariant theories, comprised of diffeomorphisms of the boundary, $\operatorname{SL}(2, \mathbb{R})$ transformations of the normal plane, and, in some cases, normal shearing transformations. We also show that if boundary conditions are chosen such that surface translations are symmetries, the algebra acquires a central extension.

KeYwords: Classical Theories of Gravity, Models of Quantum Gravity

ARXiv EPRINT: 1706.05061 


\section{Contents}

1 Introduction 1

2 Covariant canonical formalism 4

$\begin{array}{lll}3 & \text { Edge mode fields } & 7\end{array}$

$\begin{array}{lll}4 & \text { Extended phase space } & 10\end{array}$

5 JKM ambiguities $\quad 14$

$\begin{array}{lll}5.1 & L \text { ambiguity } & 15\end{array}$

$\begin{array}{lll}5.2 \theta \text { ambiguity } & 16\end{array}$

$\begin{array}{lll}5.3 Q_{\xi} \text { ambiguity } & 16\end{array}$

$\begin{array}{lll}6 & \text { Surface symmetry algebra } & 17\end{array}$

$\begin{array}{lll}6.1 & \text { Surface translations } & 21\end{array}$

$\begin{array}{lll}7 & \text { Discussion } & 23\end{array}$

$\begin{array}{ll}\text { A List of identities } & 26\end{array}$

B Edge mode derivatives in the symplectic form 31

\section{Introduction}

In gravitational theories, the problem of defining local subregions and observables is complicated by diffeomorphism invariance. Because it is a gauge symmetry, diffeomorphism invariance leads to constraints that must be satisfied by initial data for the field equations. These constraints relate the values of fields in one subregion of a Cauchy slice to their values elsewhere, so that the fields cannot be interpreted as observables localized to a particular region. While this is true in any gauge theory, a further challenge for diffeomorphism-invariant theories is that specifying a particular subregion is nontrivial, since diffeomorphisms can change the subregion's coordinate position.

A related issue in quantum gravitational theories is the problem of defining entanglement entropy for a subregion. The usual definition of entanglement entropy assumes a factorization of the Hilbert space $\mathcal{H}=\mathcal{H}_{A} \otimes \mathcal{H}_{\bar{A}}$ into tensor factors $\mathcal{H}_{A}$ and $\mathcal{H}_{\bar{A}}$ associated with a subregion $A$ and its complement $\bar{A}$. However, all physical states in a gauge theory are required to be annihilated by the constraints, and the nonlocal relations the constraints 
impose on the physical Hilbert space prevents such a factorization from occurring. ${ }^{1}$ One way of handling this nonfactorization is to define the entropy in terms of the algebra of observables for the local subregion [1]. This necessitates a choice of center for the algebra, which roughly corresponds to Wilson lines that are cut by the entangling surface. This procedure is further complicated in gravitational theories, since the local subregion and its algebra of observables must be defined in a diffeomorphism-invariant manner. Thus, the issues of local observables and entanglement in gravitational theories are intertwined.

Despite these challenges, there are indications that a well-defined notion of local observables and entanglement should exist in gravitational theories. Holography provides a compelling example, where the entanglement of bulk regions bounded by an extremal surface may be expressed in terms of entanglement in the CFT via the Ryu-Takayanagi formula and its quantum corrections $[2,3]$. Such regions are defined relationally relative to a fixed region on the boundary, and hence give a diffeomorphism-invariant characterization of the local subregion. Work regarding bulk reconstruction suggests that the algebra of observables for this subregion is fully expressible in terms of the subregion algebra of the CFT [4-9].

In addition, there are various pieces of circumstantial evidence suggesting that entanglement entropy is a well-defined and useful concept in quantum gravity. The gravitational field equations have been shown to follow from applying the first law of entanglement entropy $[10,11]$ to subregions, both in holography [12-16] and for more general gravitational theories [17-20], all of which is predicated on a well-defined notion for entanglement for the local subregion. In fact, it is conjectured that connectivity of the spacetime manifold arises from entanglement between the microscopic degrees of freedom from which the gravitational theory emerges [21]. Furthermore, entanglement entropy provides a natural explanation for the proportionality between black hole entropy and horizon area [22-25], while finessing the issue of entanglement divergences through renormalization of the gravitational couplings [26-28]. However, in the case of gauge theories, the matching between entanglement entropy divergences and the renormalization of gravitational couplings is subtle. The entropy computed using conical methods [29] contains contact terms [30-32], which are related to the presence of edge modes on the entangling surface. These arise as a consequence of the nonfactorization of the Hilbert space due to the gauge constraints. Only when the entanglement from these edge modes is properly handled does the black hole entropy have a statistical interpretation in terms of a von Neumann entropy [33-35].

Recently, Donnelly and Freidel presented a continuum description of the edge modes that arise both in Yang-Mills theory and general relativity [36]. Using covariant phase space techniques [37-40], they construct a symplectic potential and symplectic form associated with a local subregion. These are expressed as local integrals of the fields and their variations over a Cauchy surface $\Sigma$. However, one finds that they are not fully gaugeinvariant: gauge transformations that are nonvanishing at the boundary $\partial \Sigma$ change the symplectic form by boundary terms. Invariance is restored by introducing new fields in

\footnotetext{
${ }^{1}$ Strictly speaking, the factorization of a Hilbert space of any continuum field theory is formal, and only makes sense after regulating, e.g. with a lattice. The nonfactorization due to gauge constraints is more fundamental, and persists even in the regulated theory.
} 
a neighborhood of the boundary, whose change under gauge transformations cancels the boundary term from the original symplectic form. These new edge modes thus realize the idea that boundaries break gauge invariance, and cause some would-be gauge modes to become degrees of freedom associated with the subregion [41, 42].

The analysis of diffeomorphism-invariant theories in [36] was restricted to general relativity with vanishing cosmological constant. However, the construction can be generalized to arbitrary diffeomorphism-invariant theories, and it is the purpose of the present work to show how this is done. The symplectic potential for the edge modes can be expressed in terms of the Noether charge and the on-shell Lagrangian of the theory, and the symplectic form derived from it has contributions from the edge modes only at the boundary. These edge modes come equipped with set of symmetry transformations, and the symmetry algebra is represented on the phase space as a Poisson bracket algebra. The generators of the surface symmetries are given by the Noether charges associated with the transformations. We find that for generic diffeomorphism-invariant theories, the transformations that preserve the entangling surface generate the algebra $\operatorname{Diff}(\partial \Sigma) \ltimes\left(\operatorname{SL}(2, \mathbb{R}) \ltimes \mathbb{R}^{2 \cdot(d-2)}\right)^{\partial \Sigma}$. In certain cases, including general relativity, the algebra is reduced to $\operatorname{Diff}(\partial \Sigma) \ltimes \operatorname{SL}(2, \mathbb{R})^{\partial \Sigma}$, consistent with the results of [36]. Furthermore, for any other theory, there always exists a modification of the symplectic structure in the form of a Noether charge ambiguity [43] that reduces the algebra down to $\operatorname{Diff}(\partial \Sigma) \ltimes \mathrm{SL}(2, \mathbb{R})^{\partial \Sigma}$. We also discuss what happens when the algebra is enlarged to include surface translations, the transformations that do not map $\partial \Sigma$ to itself. In order for these transformations to be Hamiltonian, the dynamical fields generically have to satisfy boundary conditions at $\partial \Sigma$. Assuming the appropriate boundary conditions can be found, the full surface symmetry algebra is a central extension of either $\operatorname{Diff}(\partial \Sigma) \ltimes\left(\mathrm{SL}(2, \mathbb{R}) \ltimes \mathbb{R}^{2}\right)^{\partial \Sigma}$, or a larger, simple Lie algebra. The appearance of central charges in these algebras is familiar from similar constructions involving edge modes at asymptotic infinity or black hole horizons [42, 44, 45].

The construction of the extended phase space for arbitrary diffeomorphism-invariant theories is useful for a number of reasons. For one, higher curvature corrections to the Einstein-Hilbert action generically appear due to quantum gravitational effects. It is useful to have a formalism that can compute the corrections to the edge mode entanglement coming from these higher curvature terms. Additionally, there are several diffeomorphisminvariant theories that are simpler than general relativity in four dimensions, such as 2 dimensional dilaton gravity or 3 dimensional gravity in Anti-de-Sitter space. These could be useful testing grounds in which to understand the edge mode entanglement entropy, before trying to tackle the problem in four or higher dimensions. Finally, the general construction clarifies the relation of the extended phase space to the Wald formalism [46, 47], a connection that was also noted in [48].

This paper begins with a review of the covariant canonical formalism in section 2 . Care is taken to describe vectors and differential forms on this infinite-dimensional space, and also to understand the effect of diffeomorphisms of the spacetime manifold on the covariant phase space. Section 3 discusses the $X$ fields that appear in the extended phase space, which give rise to the edge modes. Following this, the construction of the extended phase space is given in section 4 , which describes how the edge mode fields contribute to the 
extended symplectic form. Ambiguities in the construction are characterized in section 5, and the surface symmetry algebra is identified in section 6 . Section 7 gives a summary of results and ideas for future work.

\section{Covariant canonical formalism}

The covariant canonical formalism [37-40] provides a Hamiltonian description of a field theory's degrees of freedom while maintaining spacetime covariance. This is achieved by working with the space $\mathcal{S}$ of solutions to the field equations. As long as the field equations admit a well-posed initial value formulation, each solution is in one-to-one correspondence with its initial data on some Cauchy slice. $\mathcal{S}$ may therefore be used to construct a phase space that is equivalent to Hamiltonian formalisms coordinatized by initial positions and momenta. Since a solution need not refer to a choice of initial Cauchy slice and decomposition into spatial and time coordinates, spacetime covariance remains manifest in a phase space constructed from $\mathcal{S}$. The specification of a Cauchy surface and time variable can be viewed as a choice of coordinates on $\mathcal{S}$, with each solution being identified by its initial data.

An important subtlety in this construction occurs for field equations with gauge symmetry. The space $\mathcal{S}$ involves all solutions to the field equations, so, in particular, treats two solutions that differ only by a gauge transformation as distinct. ${ }^{2}$ In this case, $\mathcal{S}$ is too large to be the correct phase space for the theory, since gauge-related solutions should represent physically equivalent configurations. Instead, the true phase space $\mathcal{P}$ should be obtained by quotienting $\mathcal{S}$ by the action of the gauge group. It is useful to view $\mathcal{S}$ as a fiber bundle, with each fiber consisting of all solutions related to each other by a gauge transformation, in which case $\mathcal{P}$ is simply the base space of this fiber bundle. As discussed in section 4, the Lagrangian for the theory imbues $\mathcal{S}$ with the structure of a presymplectic manifold, equipped with a degenerate presymplectic form. This degeneracy is necessary in order for it to project to a well-defined symplectic form on $\mathcal{P}$. The remainder of this section is devoted to describing the geometry of the space $\mathcal{S}$, while the requirements for various functions and forms (including the presymplectic form) to descend to well-defined objects on $\mathcal{P}$ are discussed in section 3 .

Working directly with $\mathcal{S}$ allows coordinate-free techniques to be applied to both the spacetime manifold and the solution space itself. In particular, the exterior calculus on the $\mathcal{S}$ gives a powerful language for describing the phase space symplectic geometry. We will follow the treatment of the exterior calculus given in $[36],{ }^{3}$ where it was used to provide an extremely efficient way of identifying edge modes for a local subregion in a gauge theory. This section provides a review of the formalism, on which the remainder of this paper heavily relies.

The theories under consideration consist of dynamical fields, including the metric $g_{a b}$ and any matter fields, propagating on a spacetime manifold $M$. These fields satisfy

\footnotetext{
${ }^{2}$ Identifying solutions with initial data is still possible if one supplements the original field equations with suitable gauge-fixing conditions. One could therefore consider $\mathcal{S}$ as being coordinatized by initial data along with a choice of gauge.

${ }^{3}$ For an extended review of this formalism, see [49] and references therein.
} 
diffeomorphism-invariant equations of motion, and the phase space is constructed from the infinite-dimensional space of solutions to these equations, $\mathcal{S}$. Despite being infinitedimensional, many concepts from finite-dimensional differential geometry, such as vector fields, one-forms, and Lie derivatives, extend straightforwardly to $\mathcal{S}$, assuming it satisfies some technical requirements such as being a Banach manifold [50, 51]. One begins by understanding the functions on $\mathcal{S}$, a wide class of which is provided by the dynamical fields themselves. Given a spacetime point $x \in M$ and a field $\phi$, the function $\phi^{x}$ associates to each solution the value of $\phi(x)$ in that solution. More generally, functionals of the dynamical fields, such as integrals over regions of spacetime, also define functions on $\mathcal{S}$ by simply evaluating the functional in a given solution. We will often denote $\phi^{x}$ simply by $\phi$, with the dependence on the spacetime point $x$ implicit.

A vector at a point of $\mathcal{S}$ describes an infinitesimal displacement away from a particular solution, and hence corresponds to a solution of the linearized field equations. Specifying a linearized solution about each full solution then defines a vector field $V$ on all of $\mathcal{S}$. The vector field acts on $\mathcal{S}$-functions as a directional derivative, and in particular its action on the functions $\phi^{x}$ is to give a new function $\Phi_{V}^{x} \equiv V\left[\phi^{x}\right]$, which, given a solution, evaluates the linearization $\Phi$ of the field $\phi$ at the point $x$. This also allows us to define the exterior derivative of the functions $\phi^{x}$, denoted $\delta \phi^{x}$. When contracted with the vector field $V$, the one-form $\delta \phi^{x}$ simply returns the scalar function $\Phi_{V}^{x}$. The one-forms $\delta \phi^{x}$ form an overcomplete basis, so that arbitrary one-forms may be expressed as sums (or integrals over the spacetime point $x$ ) of $\delta \phi^{x}$. This basis is overcomplete because the functions $\phi^{x}$ at different points $x$ are related through the equations of motion, so that the forms $\delta \phi^{x}$ are related as well.

Forms of higher degree can be constructed from the $\delta \phi^{x}$ one-forms by taking exterior products. The exterior product of a $p$-form $\alpha$ and a $q$-form $\beta$ is simply written $\alpha \beta$, and satisfies $\alpha \beta=(-1)^{p q} \beta \alpha$. Since we only ever deal with exterior products of forms defined on $\mathcal{S}$ instead of more general tensor products, no ambiguity arises by omitting the $\wedge$ symbol, which we instead reserve for spacetime exterior products. The action of the exterior derivative on arbitrary forms is fixed as usual by its action on scalar functions, along with the requirements of linearity, nilpotency $\delta^{2}=0$, and that it acts as an antiderivation,

$$
\delta(\alpha \beta)=(\delta \alpha) \beta+(-1)^{p} \alpha \delta \beta .
$$

The exterior derivative $\delta$ always increases the degree of the form by one. On the other hand, each vector field $V$ defines an antiderivation $I_{V}$ that reduces the degree by one through contraction. $I_{V}$ can be completely characterized by its action on one-forms $I_{V} \delta \phi^{x}=\Phi_{V}^{x}$, along with the antiderivation property, linearity, nilpotency $I_{\Phi}^{2}=0$, and requiring that it annihilate scalars. Just as in finite dimensions, the action of the $\mathcal{S}$ Lie derivative, denoted $L_{V}$, is related to $\delta$ and $I_{V}$ via Cartan's magic formula [51]

$$
L_{V}=I_{V} \delta+\delta I_{V}
$$

$L_{V}$ is a derivation, $L_{V}(\alpha \beta)=\left(L_{V} \alpha\right) \beta+\alpha L_{V} \beta$, that preserves the degree of the form.

We next discuss the consequences of working with diffeomorphism invariant theories. A diffeomorphism $Y$ is a smooth, invertible map, $Y: M \rightarrow M$, sending the spacetime 
manifold $M$ to itself. The diffeomorphism induces a map of tensors at $Y(x)$ to tensors at $x$ through the pullback $Y^{*}[52]$. Diffeomorphism invariance is simply the statement that if a configuration of tensor fields $\phi$ satisfy the equations of motion, then so do the pulled back fields $Y^{*} \phi$. Now consider a one-parameter family of diffeomorphisms $Y_{\lambda}$, with $Y_{0}$ the identity. This yields a family of fields $Y_{\lambda}^{*} \phi$ that all satisfy the equations of motion. The first order change induced by $Y_{\lambda}^{*}$ defines the spacetime Lie derivative $£_{\xi}$ with respect to $\xi^{a}$, the tangent vector to the flow of $Y_{\lambda}$. Consequently, $£_{\xi} \phi$ must be a solution to the linearized field equations, and the infinitesimal diffeomorphism generated by $\xi^{a}$ defines a vector field on $\mathcal{S}$, which we denote $\hat{\xi}$, whose action on $\delta \phi$ is

$$
I_{\hat{\xi}} \delta \phi \equiv £_{\xi} \phi
$$

The diffeomorphisms we have considered so far have been taken to act the same on all solutions. A useful generalization of this are the solution-dependent diffeomorphisms, defined through a function, $\mathscr{Y}: \mathcal{S} \rightarrow \operatorname{Diff}(M)$, valued in the diffeomorphism group of the manifold, $\operatorname{Diff}(M)$. Letting $Y$ denote the image of this function, we would like to understand how the Lie derivative $L_{V}$ and exterior derivative $\delta$ on $\mathcal{S}$ combine with the action of the pullback $Y^{*}$. In the case $\mathscr{Y}$ is constant on $\mathcal{S}$, the Lie derivative simply commutes with $Y^{*}$, and so $L_{V} Y^{*} \alpha=Y^{*} L_{V} \alpha$, where $\alpha$ is any form constructed from fields and their variations at a single spacetime point. When $Y$ is not constant, $V$ generates one-parameter families of diffeomorphisms $Y_{\lambda}$ and forms $\alpha_{\lambda}$ along the flow in $\mathcal{S}$. At a given solution $s_{0}$, define a solution-independent diffeomorphism $Y_{0} \equiv \mathscr{Y}\left(s_{0}\right)$ by the value of $\mathscr{Y}$ at $s_{0}$. Then $Y_{\lambda}^{*} \alpha_{\lambda}$ and $Y_{0}^{*} \alpha_{\lambda}$ are related to each other at all values of $\lambda$ by a diffeomorphism, $Y_{\lambda}^{*}\left(Y_{0}^{-1}\right)^{*}$. The first order change in these quantities at $\lambda=0$ is given by $L_{V}$, and since the two quantities differ at first order by an infinitesimal diffeomorphism, we find

$$
L_{V} Y^{*} \alpha=L_{V} Y_{0}^{*} \alpha+Y^{*} £_{\chi(Y ; V)} \alpha=Y^{*}\left(L_{V} \alpha+£_{\chi(Y ; V)} \alpha\right) .
$$

It is argued in appendix A, identity A.3, that the vector $\chi^{a}(Y ; V)$ depends linearly on $V$, and hence defines a one-form on $\mathcal{S}$, denoted $\chi_{Y}^{a} \cdot{ }^{4}$ This yields the pullback formula for $L_{V}$,

$$
L_{V} Y^{*} \alpha=Y^{*}\left(L_{V} \alpha+£_{I_{V} \chi_{Y}} \alpha\right) .
$$

Applying (2.2) to this equation, one can derive the pullback formula for exterior derivatives from [36] (see A.5 for details),

$$
\delta Y^{*} \alpha=Y^{*}\left(\delta \alpha+£ \chi_{Y} \alpha\right) .
$$

A number of properties of the variational vector field $\chi_{Y}^{a}$ follow from the formulas above. First, note $\chi_{Y}^{a}$ is not an exact form on $\mathcal{S}$; rather, its exterior derivative can be deduced from (2.6),

$$
0=\delta \delta Y^{*} \alpha=Y^{*}\left(\delta £ \chi_{Y} \alpha+£ \chi_{Y} \delta \alpha+£ \chi_{Y} £ \chi_{Y} \alpha\right)=Y^{*}\left(£_{\delta\left(\chi_{Y}\right)} \alpha+£ \chi_{Y} £ \chi_{Y} \alpha\right),
$$

\footnotetext{
${ }^{4}$ In [36], $\chi_{Y}^{a}$ was denoted $\delta_{Y}^{a}$. We choose a different notation to emphasize that $\chi_{Y}^{a}$ is not an exact form, and to avoid confusion with the exterior derivative $\delta$.
} 
and applying A.7, we conclude

$$
\delta\left(\chi_{Y}\right)^{a}=-\frac{1}{2}\left[\chi_{Y}, \chi_{Y}\right]^{a} .
$$

Another useful formula relates $\chi_{Y}^{a}$ to the vector $\chi_{Y^{-1}}^{a}$ associated with the inverse of $Y$. Using that $Y^{*}$ and $\left(Y^{-1}\right)^{*}$ are inverses of each other, we find

$$
\delta \alpha=\delta Y^{*}\left(Y^{-1}\right)^{*} \alpha=Y^{*}\left[\delta\left(Y^{-1}\right)^{*} \alpha+£_{\chi_{Y}}\left(Y^{-1}\right)^{*} \alpha\right]=\delta \alpha+£_{\delta_{Y^{-1}}} \alpha+£_{Y^{*} \chi_{Y}} \alpha,
$$

where the last equality involves the identity A.8. This implies

$$
\chi_{Y^{-1}}^{a}=-Y^{*} \chi_{Y}^{a} .
$$

Additional identities are derived in appendix A.

Finally, as a spacetime vector field, $\chi_{Y}^{a}$ also defines a vector-valued one-form $\hat{\chi}_{Y}$ on $\mathcal{S}$, which acts as $I_{\hat{\chi}_{Y}} \delta \phi=£ \chi_{Y} \phi$. The contraction $I_{\hat{\chi}_{Y}}$ defines a derivation that preserves the degree of the form, in contrast to $I_{\hat{\xi}}$, which is an antiderivation that reduces the degree. Similarly, $\delta\left(\chi_{Y}\right)^{a}$ defines a vector-valued two-form on $\mathcal{S}$, and produces an antiderivation $I_{\delta\left(\chi_{Y}\right)^{\wedge}}$ that increments the degree.

\section{Edge mode fields}

Edge modes appear when a gauge symmetry is broken due to the presence of a boundary $\partial \Sigma$ of a Cauchy surface $\Sigma$. The classical phase space or quantum mechanical Hilbert space associated with $\Sigma$ transforms nontrivially under gauge transformations that act at the boundary. This can be understood from the perspective of Wilson loops that are cut by the boundary. A closed Wilson loop is gauge-invariant, but the cut Wilson loop becomes a Wilson line in $\Sigma$, whose endpoints transform in some representation of the gauge group. To account for these cut-Wilson-loop degrees of freedom, one can introduce fictitious charged fields at $\partial \Sigma$, which can be attached to the ends of the Wilson lines to produce a gaugeinvariant object. These new fields are the edge modes of the local subregion. They account for the possibility of charge density existing outside of $\Sigma$, which would affect the fields in $\Sigma$ due to Gauss law constraints. The contribution of the edge modes to the entanglement can therefore be interpreted as parameterizing ignorance of such localized charge densities away from $\Sigma$.

A similar picture arises in the classical phase space of a diffeomorphism-invariant theory. The edge modes appear when attempting to construct a symplectic structure associated with $\Sigma$ for the solution space $\mathcal{S}$. Starting with the Lagrangian of the theory, one can construct from its variations a symplectic current $\omega$, a spacetime $(d-1)$-form whose integral over a spatial subregion $\Sigma$ provides a candidate presymplectic form. However, this form fails to be diffeomorphism invariant for two reasons. First, a diffeomorphism moves points on the mainfold around, and hence changes the shape and coordinate location of the surface. Second, since solutions related to each other by a diffeomorphism represent the same physical configuration, the true phase space $\mathcal{P}$ is obtained by projecting all solutions in a gauge orbit in $\mathcal{S}$ down to a single representative. In order for the symplectic 
form to be compatible with this projection, the infinitesimal diffeomorphisms must be degenerate directions of the presymplectic form [50]. ${ }^{5}$ This is equivalent to saying that the Hamiltonian generating the diffeomorphism may be chosen to vanish. While the symplectic form obtained by integrating $\omega$ over a surface is degenerate for diffeomorphisms that vanish sufficiently quickly at its boundary, those that do not produce boundary terms that spoil degeneracy.

The problem of non-invariance due to diffeomorphisms that move the surface is solved by defining the surface's location in a diffeomorphism-invariant manner. There are a variety of ways that this can be done. One example comes from the Ryu-Takayanagi prescription in holography, where the bulk entangling surface $\partial \Sigma$ is defined as the extremal surface that asymptotes to a given subregion on the boundary of AdS [2]. Another set of techniques are the relational constructions of [53], where one set of fields can be used to define a coordinate system, and subregions can be defined relationally to these coordinate fields. An important point about the edge modes is that they are necessary even after dealing with this first source of non-invariance: the presymplectic form may still not be appropriately degenerate even after specifying the subregion invariantly. The remainder of this work will primarily be focused on how this second issue is resolved, although the extended phase space provides a formal solution to the first issue as well.

As demonstrated in [36], both problems can be handled by introducing a collection of additional fields $X$ whose contribution to the symplectic form restores diffeomorphism invariance. These fields are the edge modes of the extended phase space. This section is devoted to describing these fields and their transformation properties under diffeomorphisms; the precise way in which they contribute to the symplectic form is discussed in section 4 .

The fields $X$ can be defined through a $\operatorname{Diff}(M)$-valued function $\mathscr{X}: \mathcal{S} \rightarrow \operatorname{Diff}(M)$. In a given solution $s, X$ is identified with the diffeomorphism in the image of the map, $X=\mathscr{X}(s)$. One way to interpret $X$ is as defining a map from (an open subset of) $\mathbb{R}^{d}$ into the spacetime manifold $M$, and hence can be thought of as a choice of coordinate system covering the local subregion $\Sigma{ }^{6}$ The problem of defining the subregion $\Sigma$ is solved by declaring it to be the image under the $X$ map of some fiducial subregion $\sigma$ in $\mathbb{R}^{d}$. A full solution to the field equations now consists of specifying the map $X$ as well as the value of the dynamical fields $\phi(x)$ at each point in spacetime. The transformation law for $X$ under a diffeomorphism $Y: M \rightarrow M$ is given by the pullback along $Y^{-1}, \bar{X}=Y^{-1} \circ X$.

\footnotetext{
${ }^{5}$ Indeed, only functions on $\mathcal{S}$ that are constant along the gauge orbits descend to well-defined functions on $\mathcal{P}$. Similarly, the only forms that survive the projection must be both constant along gauge orbits and annihilate vectors tangent to the gauge orbits. In particular, the functions $\phi^{x}$ constructed from the dynamical fields do not survive the projection, while diffeomorphism-invariant functionals of $\phi^{x}$ do survive. Note that this is one reason for working with $\mathcal{S}$ : it is technically simpler to derive relations involving the local field functions $\phi^{x}$ in $\mathcal{S}$ than always working with diffeomorphism-invariant objects in $\mathcal{P}$. Most of the relations in this paper are derived in $\mathcal{S}$, and then are argued to hold in $\mathcal{P}$ if they involve diffeomorphisminvariant functionals and are properly degenerate.

${ }^{6}$ We assume for simplicity that the subregion of interest can be covered by a single coordinate system. For topologically nontrivial subregions, the fields may consist of a collection of maps $X_{i}$, one for each coordinate patch needed to cover the region.
} 
Since $X$ defines a diffeomorphism from $\mathbb{R}^{d}$ to $M$, it can be used to pull back tensor fields on $M$ to $\mathbb{R}^{d}$. We can argue as before that the Lie derivative $L_{V}$ and exterior derivative $\delta$ satisfy pullback forumlas analogous to equations (2.4) and (2.6),

$$
\begin{aligned}
L_{V} X^{*} \alpha & =X^{*}\left(L_{V} \alpha+£_{\left.I_{V} \chi_{X} \alpha\right)}\right. \\
\delta X^{*} \alpha & =X^{*}\left(\delta \alpha+£ \chi_{X} \alpha\right),
\end{aligned}
$$

which serve as defining relations for the variational spacetime vector $\chi_{X}^{a}$. The result of contracting $\chi_{X}^{a}$ with a vector field $\hat{\xi}$ corresponding to a spacetime diffeomorphism can be deduced by first noting that the pulled back fields $X^{*} \phi$ are invariant under diffeomorphisms, since

$$
\bar{X}^{*} Y^{*} \phi=X^{*}\left(Y^{-1}\right)^{*} Y^{*} \phi=X^{*} \phi .
$$

In particular, the $\mathcal{S}$ Lie derivative $L_{\hat{\xi}}$ must annihilate $X^{*} \phi$ for any $\xi$, so from (3.1),

$$
0=L_{\hat{\xi}} X^{*} \phi=X^{*}\left(L_{\hat{\xi}} \phi+£_{I_{\hat{\xi}} \chi_{X}} \phi\right)=X^{*}\left(£_{\xi} \phi+£_{I_{\hat{\xi}}} \chi_{X} \phi\right)
$$

and hence

$$
I_{\hat{\xi}} \chi_{X}^{a}=-\xi^{a} .
$$

We can also derive the transformation law for $\chi_{X}^{a}$ under a diffeomorphism from the pullback formulas (2.6) and (3.2). On the one hand we have

$$
\delta \bar{X}^{*} \alpha=\bar{X}^{*}\left(\delta \alpha+£ \chi_{\bar{X}} \alpha\right)
$$

while on the other hand this can also be computed as

$$
\delta \bar{X}^{*} \alpha=\delta X^{*}\left(Y^{-1}\right)^{*} \alpha=X^{*}\left[\delta\left(Y^{-1}\right)^{*} \alpha+£ \chi_{X}\left(Y^{-1}\right)^{*} \alpha\right]=\bar{X}^{*}\left(\delta \alpha+£ \chi_{Y^{-1}} \alpha+£_{Y^{*} \chi_{X}} \alpha\right)
$$

where the last equality employed identity A.8. Comparing these expressions and applying the formula (2.10) for $\chi_{Y^{-1}}^{a}$ gives the transformation law

$$
\chi_{\bar{X}}^{a}=Y^{*}\left(\chi_{X}^{a}-\chi_{Y}^{a}\right)
$$

The $X$ fields lead to an easy prescription for forming diffeomorphism-invariant quantities: simply work with the pulled back fields $X^{*} \phi$. These are diffeomorphism-invariant due to equation (3.3), and consequently the variation $\delta X^{*} \phi$ is as well. We can explicitly confirm that $\delta X^{*} \phi$ are annihilated by infinitesimal diffeomorphisms $\hat{\xi}$ :

$$
I_{\hat{\xi}} \delta X^{*} \phi=I_{\hat{\xi}} X^{*}\left(\delta \phi+£ \chi_{X} \phi\right)=X^{*}\left(£_{\xi} \phi-£_{\xi} \phi\right)=0 .
$$

Note that these relations ensure that $X^{*} \phi$ and $\delta X^{*} \phi$ descend to functions on the reduced phase space $\mathcal{P}$, after quotienting $\mathcal{S}$ by the degenerate directions of the presymplectic form. Another combination of one-forms that appears frequently is $\alpha+I_{\hat{\chi}_{X}} \alpha$, and it is easily checked that $I_{\hat{\xi}}$ annihilates this sum. Finally, we note that when no confusion will arise, we will simply denote $\chi_{X}^{a}$ by $\chi^{a}$ to avoid excessive clutter. When referring to other diffeomorphisms besides $X$, we will explicitly include the subscript, as in $\chi_{Y}^{a}$. 


\section{Extended phase space}

We now turn to the problem of defining a gauge-invariant symplectic form to associate with the local subregion. In this work, the precise meaning of a local subregion is the domain of dependence of some spacelike hypersurface $\Sigma,{ }^{7}$ which serves as a Cauchy surface for the subregion. We further require that $\Sigma$ have a boundary $\partial \Sigma$, so that it may be thought of as a subspace of a larger Cauchy surface for the full spacetime. The standard procedure of $[46,47,50]$ for constructing a symplectic form for a diffeomorphism-invariant field theory begins with a Lagrangian $L[\phi]$, a spacetime $d$-form constructed covariantly from the dynamical fields $\phi$. Its variation takes the form

$$
\delta L=E \cdot \delta \phi+d \theta
$$

where $E=0$ are the dynamical field equations, and the exact form $d \theta$, where $d$ denotes the spacetime exterior derivative, defines the symplectic potential current $(d-1)$-form $\theta \equiv \theta[\phi ; \delta \phi]$, which is a one-form on solution space $\mathcal{S}$. The $\mathcal{S}$-exterior derivative of $\theta$ defines the symplectic current $(d-1)$-form, $\omega=\delta \theta$, whose integral over $\Sigma$ normally defines the presymplectic form $\Omega_{0}$ for the phase space. As a consequence of diffeomorphism-invariance, $\Omega_{0}$ contains degenerate directions: it annihilates any infinitesimal diffeomorphism generated by vector field $\xi^{a}$ that vanishes sufficiently quickly near the boundary. This is succinctly expressed for such a vector field by $I_{\hat{\xi}} \Omega_{0}=0$. The true phase space $\mathcal{P}$ is obtained by quotienting out these degenerate directions by mapping all diffeomorphism-equivalent solutions to a single point in $\mathcal{P}$. $\Omega_{0}$ then defines a nondegenerate symplectic form on $\mathcal{P}$ through the process of phase space reduction [50].

This procedure is deficient for a local subregion because $\Omega_{0}$ fails to be degenerate for diffeomorphisms that act near the Cauchy surface's boundary $\partial \Sigma$. If the boundary were at asymptotic infinity, such diffeomorphisms could be disallowed by imposing boundary conditions on the fields, or could otherwise be regarded as true time evolution with respect to the fixed asymptotic structure, in which case degeneracy would not be expected [40]. For a local subregion, however, neither option is acceptable. Imposing a boundary condition on the fields at $\partial \Sigma$ has a nontrivial effect on the dynamics [54-56], whereas we are interested in a phase space that locally reproduces the same dynamics as the theory defined on the full spacetime manifold $M$. Furthermore, the diffeomorphisms acting at $\partial \Sigma$ cannot be regarded as true time evolution generated by nonvanishing Hamiltonians, because these diffeomorphisms are degenerate directions of a presymplectic form for the entire manifold $M$.

Donnelly and Freidel [36] proposed a resolution to this issue by extending the local phase space to include the $X$ fields described in section 3. The minimal prescription for introducing them into the theory is to simply replace the Lagrangian with its pullback $X^{*} L$. Since the Lagrangian is a covariant functional of the fields, $X^{*} L[\phi]=L\left[X^{*} \phi\right]$, so

\footnotetext{
${ }^{7}$ The requirement that $\Sigma$ be spacelike is necessary in order to interpret the symplectic form constructed on it as characterizing a subset of the theory's degrees of freedom. While the construction would seem to also apply to timelike hypersurfaces, such a hypersurface has an empty domain of dependence, and so there is no sense in which it determines the dynamics in some open subset of the manifold.
} 
that the pulled back Lagrangian depends only on the redefined fields $X^{*} \phi$, and is otherwise independent of $X$. The variation of this Lagrangian gives

$$
\delta L\left[X^{*} \phi\right]=E\left[X^{*} \phi\right] \cdot \delta X^{*} \phi+d \theta\left[X^{*} \phi ; \delta X^{*} \phi\right] .
$$

Thus the redefined fields satisfy the same equations of motion $E\left[X^{*} \phi\right]=0$ as the original fields, and, due to diffeomorphism invariance, this implies that the original $\phi$ fields must satisfy the equations as well. Additionally, the Lagrangian had no further dependence on $X$, which means the $X$ fields do not satisfy any field equations. If $X$ is understood as defining a coordinate system for the local subregion, the dynamics of the extended $(\phi, X)$ system is simply given by the original field equations, expressed in an arbitrary coordinate system determined by $X$.

The symplectic potential current is read off from (4.2),

$$
\theta^{\prime}=\theta\left[X^{*} \phi ; \delta X^{*} \phi\right]=\theta\left[X^{*} \phi ; X^{*}(\delta \phi+£ \chi \phi)\right]=X^{*}\left(\theta+I_{\hat{\chi}} \theta\right) .
$$

This object is manifestly invariant with respect to solution-dependent diffeomorphisms, since both $X^{*} \phi$ and $\delta X^{*} \phi$ are. In particular, $\theta^{\prime}$ annihilates any infinitesimal diffeomorphism $I_{\hat{\xi}}$, as a consequence of the fact that $I_{\hat{\xi}} \delta X^{*} \phi=0$ (see equation (3.4)). An equivalent expression for $\theta^{\prime}$ can be obtained by introducing the Noether current for a vector field $\xi^{a}$,

$$
J_{\xi}=I_{\hat{\xi}} \theta-i_{\xi} L
$$

where $i_{\xi}$ denotes contraction with the spacetime vector $\xi^{a}$. Due to diffeomorphism invariance, $J_{\xi}$ is an exact form when the equations of motion hold $[46,47]$, and may be written

$$
J_{\xi}=d Q_{\xi}+C_{\xi}
$$

where $Q_{\xi}$ is the Noether charge and $C_{\xi}=0$ are combinations of the field equations that comprise the constraints for the theory [57]. Then $\theta^{\prime}$ in (4.3) may be expressed on-shell

$$
\theta^{\prime}=X^{*}\left(\theta+i_{\chi} L+d Q \chi\right)
$$

As an aside, note that we can vary the Lagrangian with respect to $(\phi, X)$ instead of the redefined fields $\left(X^{*} \phi, X\right)$, and equivalent dynamics arise. This variation produces

$$
\delta X^{*} L[\phi]=X^{*}(\delta L+£ \chi L)=X^{*}(E \cdot \delta \phi)+d X^{*}\left(\theta+i_{\chi} L\right),
$$

where Cartan's magic formula $£_{\chi}=i_{\chi} d+d i_{\chi}$ was used, along with the fact that $d$ commutes with pullbacks. Again, $\phi$ satisfies the same field equation $E[\phi]=0$, and $X$ is subjected to no dynamical equations. This variation suggests a potential current $\theta^{\prime \prime}=X^{*}\left(\theta+i_{\chi} L\right)$, which differs from (4.6) by the exact form $d X^{*} Q_{\chi}$. This difference is simply an ambiguity in the definition of the potential current, since shifting it by an exact form does not affect equation $(4.1)[43,47]$. However, $\theta^{\prime \prime}$ does not annihilate infinitesimal diffeomorphisms $I_{\hat{\xi}}$, making $\theta^{\prime}$ the preferred choice. The degeneracy requirement for the symplectic potential current therefore gives a prescription to partially fix its ambiguities [48], although additional ambiguities remain, and are discussed in section 5 . 
The symplectic potential $\Theta$ is now constructed by integrating $\theta^{\prime}$ over $\Sigma$. Since $\theta^{\prime}$ is defined as a pullback by $X^{*}$, its integral must be over the pre-image $\sigma$, for which $X(\sigma)=\Sigma$. This gives

$$
\begin{aligned}
\Theta & =\int_{\sigma} \theta\left[X^{*} \phi ; \delta X^{*} \phi\right] \\
& =\int_{\Sigma}\left(\theta+i_{\chi} L\right)+\int_{\partial \Sigma} Q \chi .
\end{aligned}
$$

The second line uses the alternative expression (4.6) for $\theta^{\prime}$, and is written as an integral of fields defined on the original Cauchy surface $\Sigma$, without pulling back by $X$. This makes use of the general formula $\int_{\sigma} X^{*} \alpha=\int_{X(\sigma)} \alpha$, and also applies Stoke's theorem $\int_{\Sigma} d \alpha=\int_{\partial \Sigma} \alpha$ to write the Noether charge as a boundary integral. Equation (4.9) differs from the symplectic potential for the nonextended phase space, $\Theta_{0}=\int_{\Sigma} \theta$, by both a boundary term depending on the Noether charge, as well as a bulk term coming from the on-shell value of the Lagrangian. For vacuum general relativity with no cosmological constant, this extra bulk contribution vanishes, being proportional to the Ricci scalar [36]. However, when matter is present or the cosmological constant is nonzero, this extra bulk contribution to $\Theta$ can survive. As we discuss below, this bulk term imbues the symplectic form on the reduced phase space $\mathcal{P}$ with nontrivial cohomology.

Taking an exterior derivative of $\Theta$ yields the symplectic form, $\Omega=\delta \Theta$. The expression (4.8) leads straightforwardly to

$$
\Omega=\int_{\sigma} \omega\left[X^{*} \phi ; \delta X^{*} \phi, \delta X^{*} \phi\right]
$$

where we recall the definition of the symplectic current $\omega=\delta \theta$. This expression for $\Omega$ makes it clear that it is invariant with respect to all diffeomorphisms, and that infinitesimal diffeomorphisms are degenerate directions, again because $I_{\hat{\xi}} \delta X^{*} \phi=0$. The symplectic form can also be expressed as an integral over $\Sigma$ and its boundary using the original fields $\phi$, by computing the exterior derivative of (4.9). Noting that the integrands implicitly involve a pullback by $X^{*}$, we find

$$
\Omega=\int_{\Sigma}\left(\omega+£ \chi \theta+\delta i_{\chi} L+£ \chi i_{\chi} L\right)+\int_{\partial \Sigma}\left(\delta Q_{\chi}+£ \chi Q \chi\right)
$$

The first term is the symplectic form for the nonextended theory, $\Omega_{0}=\int_{\Sigma} \omega$. The remaining three terms in the bulk $\Sigma$ integral simplify to an exact form on-shell $d\left(i_{\chi} \theta+\frac{1}{2} i_{\chi} i_{\chi} L\right.$ ) (see identity A.10), so the final expression is

$$
\Omega=\int_{\Sigma} \omega+\int_{\partial \Sigma}\left[\delta Q_{\chi}+£ \chi Q_{\chi}+i_{\chi} \theta+\frac{1}{2} i_{\chi} i_{\chi} L\right]
$$

Hence, we arrive at the important result that the symplectic form differs from $\Omega_{0}$ by terms localized on the boundary $\partial \Sigma$ involving $\chi^{a}$. This immediately implies that $\Omega$ has degenerate directions: any phase space vector field $V$ that vanishes on $\delta \phi$ and whose contraction with $\chi^{a}$ vanishes sufficiently quickly near $\partial \Sigma$ will annihilate $\Omega$. In fact, only 
the values of $\chi^{a}$ and $\nabla_{b} \chi^{a}$ at $\partial \Sigma$ contribute to (4.12); all other freedom in $\chi^{a}$ is pure gauge. To see why these are the only relevant pieces of $\chi^{a}$ for the symplectic form, we can use the explicit expression for the Noether charge given in [47]. Up to ambiguities which are discussed in section 5 , the Noether charge is given by

$$
Q_{\xi}=-\epsilon_{a b} E_{d}^{a b c} \nabla_{c} \xi^{d}+W_{c} \xi^{c}
$$

where $\epsilon_{a b}$ is the spacetime volume form with all but the first two indices suppressed, $E^{a b c d}=\frac{\delta \mathcal{L}}{\delta R_{a b c d}}$ is the variational derivative of the Lagrangian scalar $\mathcal{L}=-(* L)$ with respect to the Riemann tensor, and inherits the index symmetries of the Riemann tensor, and $W_{c}[\phi]$ is a tensor with $(d-2)$ covariant, antisymmetric indices suppressed, constructed locally from the dynamical fields; its precise form is not needed in this work.

The last two terms in (4.12) depend only on the value of $\chi^{a}$ on $\partial \Sigma$, while the terms involving $Q_{\chi}$ can depend on derivatives of $\chi^{a}$. From (4.13), $Q_{\chi}$ involves one derivative of $\chi^{a}$, and (4.12) has terms involving the derivative of $Q_{\chi}$, so that up to two derivatives of $\chi^{a}$ could contribute to the symplectic form. To see how these derivatives appear, we decompose $\delta Q \chi$ as

$$
\delta Q_{\chi}=Q_{\delta(\chi)}+\varphi_{\chi}
$$

where $\uparrow_{\xi}=q_{\xi}[\phi ; \delta \phi]^{8}$ is a variational one-form depending on a vector $\xi$ (which can be a differential form on $\mathcal{S}$ ), given by

$$
१_{\xi}=-\delta\left(\epsilon_{a b} E_{d}^{a b c}\right) \nabla_{c} \xi^{d}-\epsilon_{a b} E_{d}^{a b c} \delta \Gamma_{c e}^{d} \xi^{e}+\delta W_{c} \xi^{c},
$$

and $\delta \Gamma_{c e}^{d}$ is the variation of the Christoffel symbol,

$$
\delta \Gamma_{c e}^{d}=\frac{1}{2} g^{d f}\left(\nabla_{c} \delta g_{f e}+\nabla_{e} \delta g_{f c}-\nabla_{f} \delta g_{c e}\right) .
$$

This decomposition is useful because ${ }^{\chi} \chi$ contains only first derivatives of $\chi^{a}$, while $Q_{\delta \chi}=$ $-\frac{1}{2} Q_{[\chi, \chi]}$ involves second derivatives through the derivative of the vector field Lie bracket.

In appendix B, it is argued that the second derivatives of $\chi^{a}$ in $Q_{\delta(\chi)}+£_{\chi} Q_{\chi}$ cancel out, so that the boundary contribution in (4.12) depends on only $\chi^{a}$ and $\nabla_{b} \chi^{a}$ at $\partial \Sigma$. This means that $\Omega$ has a large number of degenerate directions, corresponding to all values of $\chi^{a}$ on $\Sigma$ that are not fixed by the values of $\chi^{a}$ and $\nabla_{b} \chi^{a}$ at the boundary. The true phase space $\mathcal{P}$ is then obtained by quotienting out these pure gauge degrees of freedom. In doing so, $\Omega$ descends to a nondegenerate, closed two-form on the quotient space [50]. However, the symplectic potential $\Theta$ does not survive this projection. It depends nontrivially on the value of $\chi^{a}$ everywhere on $\Sigma$ through the term involving the Lagrangian in (4.9), which causes it to become a multivalued form on the quotient space. One way to see its multivaluedness is to note that $i_{\chi} L$ is a top rank form on $\Sigma$, so, by the Poincaré lemma applied to $\Sigma$, it can be expressed as the exterior derivative of a $(d-2)$-form,

$$
\left.i_{\chi} L\right|_{\Sigma}=d h_{X} i_{\chi} L
$$

\footnotetext{
${ }^{8} \mathrm{Q}$ is the archaic Greek letter "qoppa."
} 
Here, $h_{X}$ is the homotopy operator that inverts the exterior derivative $d$ on closed forms on $\Sigma$ [58]. As the notation suggests, it depends explicitly on the value of the $X$ fields throughout $\Sigma$, which we recall can be thought of as defining a coordinate system for the subregion. Since $h_{X} i_{\chi} L$ is a spacetime $(d-2)$-form and an $\mathcal{S}$ one-form, evaluated at $\partial \Sigma$ it may be expressed in terms of $\chi^{a}$ and $\delta \phi$ at $\partial \Sigma$, which provide a basis for local variational forms. Hence,

$$
\int_{\Sigma} i_{\chi} L=\int_{\partial \Sigma} h_{X} i_{\chi} L
$$

and we see that this latter expression depends on $\chi^{a}$ at $\partial \Sigma$, so therefore will project to the quotient space. However, $h_{X}$ will be a different operator depending on the values of the $X$ fields on $\Sigma$, and hence this boundary integral will give a different form on the reduced phase space for different bulk values of $X$. This shows that the Lagrangian term in $\Theta$ projects to a multivalued form on the quotient space.

The failure of $\Theta$ to be single-valued implies that the reduced phase space $\mathcal{P}$ has nontrivial cohomology. In particular, the projected symplectic form $\Omega$ is not exact, despite being closed. For a given choice of the value of $\Theta$, the equation $\Omega=\delta \Theta$ still holds locally near a given solution in the reduced phase space, but there can be global obstructions since $\Theta$ may not return to the same value after tracing out a closed loop in the solution space. It would be interesting to investigate the consequences of this nontrivial topology of the reduced phase space, and in particular whether it has any relation to the appearance of central charges in the surface symmetry algebra.

Finally, note that for vacuum general relativity with no cosmological constant, the Lagrangian vanishes on shell, being proportional to the Ricci scalar. In this special case, $\Theta$ is not multivalued and descends to a well-defined one-form on the reduced phase space, suggesting that the phase space topology simplifies. However, the inclusion of a cosmological constant or the presence of matter anywhere in the local subregion leads back to the generic case in which $\Theta$ is multivalued.

\section{$5 \quad$ JKM ambiguities}

The constructions of the symplectic potential current $\theta$ and Noether charge $Q_{\xi}$ are subject to a number of ambiguities identified by Jacobson, Kang and Myers (JKM) [43, 47]. These ambiguities correspond to the ability to add an exact form to the Lagrangian $L$, the potential current $\theta$, or the Noether charge $Q_{\xi}$ without affecting the dynamics or the defining properties of these forms. Normally it is required that the ambiguous terms be locally constructed from the dynamical fields in a spacetime-covariant manner. In the extended phase space, however, there is additional freedom provided by the $X$ fields as well as the surfaces $\Sigma$ and $\partial \Sigma$ to construct forms that would otherwise fail to be covariant. The freedom provided by the $X$ fields is considerable, given that they can be used to construct homotopy operators as in (4.17) and (4.18) that mix the local dynamical fields $\phi$ at different spacetime points. For this reason, we refrain from using the $X$ fields in such an explicit manner to construct ambiguity terms. However, we allow for ambiguity terms that are constructed using the structures provided by $\Sigma$ and $\partial \Sigma$, such as their induced metrics and 
extrinsic curvatures. This allows for a wider class of Noether charges, including those that appear in holographic entropy functionals and the second law of black hole mechanics for higher curvature theories [59-62].

A simple example of which types of objects are permitted in constructing the ambiguity terms is provided by the unit normal $u_{a}$ to $\Sigma$ versus the lapse function $N$. Interpreting $X^{\mu}$ as a coordinate system for the local subregion, we can take $\Sigma$ to lie at $X^{0}=0$. Then the lapse and unit normal are related by

$$
u_{a}=-N \nabla_{a} X^{0} .
$$

The form $\nabla_{a} X^{0}$ depends explicitly on the $X$ field, and hence is not allowed in our constructions. However, the unit normal $u_{a}$ can be constructed using only the surface $\Sigma$ and the metric, and hence is independent of the $X$ fields. This then implies that $N$ also depends on the $X$ fields, and so the lapse function cannot explicitly be used in constructing ambiguity terms.

\section{$5.1 \quad L$ ambiguity}

The first ambiguity corresponds to adding an exact form $d \alpha$ to the Lagrangian. This does not affect the equations of motion; however, its variation now contributes to $\theta$. The following changes occur from adding this term to the Lagrangian:

$$
\begin{aligned}
L & \rightarrow L+d \alpha \\
\theta & \rightarrow \theta+\delta \alpha \\
J_{\xi} & \rightarrow J_{\xi}+d i_{\xi} \alpha \\
Q_{\xi} & \rightarrow Q_{\xi}+i_{\xi} \alpha .
\end{aligned}
$$

Note that since $\theta$ changes by an $\mathcal{S}$-exact form, the symplectic current $\omega$ is unaffected. Incorporating these changes into the definition of the symplectic potential (4.9) changes $\Theta$ by

$$
\Theta \rightarrow \Theta+\int_{\Sigma}\left(\delta \alpha+i_{\chi} d \alpha\right)+\int_{\partial \Sigma} i_{\chi} \alpha=\Theta+\delta \int_{\Sigma} \alpha .
$$

We point out that the new term annihilates infinitesimal diffeomorphisms $I_{\hat{\xi}}$, so that $\Theta$ remains fully diffeomorphism-invariant. Since $\Theta$ changes by an $\mathcal{S}$-exact form, the symplectic form $\Omega=\delta \Theta$ receives no change from this type of ambiguity, which can also be checked by tracking the changes of all quantities in (4.12). Given that only $\Omega$, and not $\Theta$, is needed in the construction of the phase space, this ambiguity in $L$ has no effect on the phase space. However, it has some relevance to the surface symmetry algebra discussed in section 6 . The generators of this algebra are given by the Noether charge, and for surface symmetries that move $\partial \Sigma$ (the "surface translations"), this ambiguity would appear to have an effect. However, as discussed in subsection 6.1, once the appropriate boundary terms are included in the generators, the result is independent of this ambiguity. The form of the generator does motivate a natural prescription for fixing the ambiguity such that the Lagrangian has a well-defined variational principle, so that it is completely stationary on-shell, as opposed to being stationary up to boundary contributions. 


\section{$5.2 \theta$ ambiguity}

The second ambiguity comes from the freedom to add an exact form $d \beta$ to $\theta$, since doing so does not affect its defining equation (4.1). Here, $\beta \equiv \beta[\phi ; \delta \phi]$ is a spacetime $(d-2)$-form and a one-form on $\mathcal{S}$. The changes that arise from this addition are

$$
\begin{aligned}
\theta & \rightarrow \theta+d \beta \\
\omega & \rightarrow \omega+d \delta \beta \\
J_{\xi} & \rightarrow J_{\xi}+d I_{\hat{\xi}} \beta \\
Q_{\xi} & \rightarrow Q_{\xi}+I_{\hat{\xi}} \beta .
\end{aligned}
$$

Under these transformations, the symplectic potential (4.9) changes to

$$
\Theta \rightarrow \Theta+\int_{\partial \Sigma}\left(\beta+I_{\hat{\chi}} \beta\right)
$$

Hence, the symplectic potential is modified by an arbitrary boundary term $\beta$, accompanied by $I_{\hat{\chi}} \beta$ that ensures that $\Theta$ retains degenerate directions along linearized diffeomorphisms. Unlike the $L$ ambiguity, this modification is not $\mathcal{S}$-exact, and changes the boundary terms in the symplectic form,

$$
\Omega \rightarrow \Omega+\int_{\partial \Sigma}\left(\delta \beta+\delta I_{\hat{\chi}} \beta+£ \chi \beta+£ \chi I_{\hat{\chi}} \beta\right) .
$$

Because $\beta$ can in principle involve arbitrarily many derivatives of $\delta \phi$, its presence can cause $\Omega$ to depend on second or higher derivatives of $\chi^{a}$ on the boundary. This affects which parts of $\chi^{a}$ correspond to degenerate directions, and will lead to different numbers of boundary degrees of freedom in the reduced phase space. As discussed in section 6, this ambiguity can also be used to reduce the surface symmetry algebra to a subalgebra.

Give that $\beta$ contributes to $\Theta$ and $\Omega$ only at the boundary, it can involve tensors associated with the surface $\partial \Sigma$ that do not correspond to spacetime-covariant tensors, such as the extrinsic curvature. This allows the Dong entropy [59-61], which differs from the Wald entropy $[46,47]$ by extrinsic curvature terms, to be viewed as a Noether charge with a specific choice of ambiguity terms. This is the point of view advocated for in [62], where the ambiguity was resolved by requiring that the entropy functional derived from the resultant Noether charge satisfy a linearized second law. In general, fixing the ambiguity requires some additional input, motivated by the particular application at hand.

\section{$5.3 Q_{\xi}$ ambiguity}

The final ambiguity is the ability to shift $Q_{\xi}$ by a closed form $\gamma$, with $d \gamma=0$. Since $Q_{\xi}$ depends linearly on $\xi^{a}$ and its derivatives, $\gamma$ should be chosen to also satisfy this requirement. If $\gamma$ is identically closed for all $\xi^{a}$, it then follows that it must be exact, $\gamma=d \nu$ [63]. Its integral over the closed surface $\partial \Sigma$ then vanishes, so that it has no effect on $\Theta$ or $\Omega$. 


\section{$6 \quad$ Surface symmetry algebra}

The extended phase space constructed in section 4 contains new edge mode fields $\chi^{a}$ on the boundary of the Cauchy surface for the local subregion, whose presence is required in order to have a gauge-invariant symplectic form. Associated with the edge modes are a new class of transformations that leave the symplectic form and the equations of motion invariant. These new transformations comprise the surface symmetry algebra. This algebra plays an important role in the quantum theory when describing the edge mode contribution to the entanglement entropy, thus it is necessary to identify the algebra and its canonical generators.

As discussed in [36], the surface symmetries coincide with diffeomorphisms in the preimage space, $Z: \mathbb{R}^{d} \rightarrow \mathbb{R}^{d}$, where $\mathbb{R}^{d} \supset X^{-1}(M)$. These leave the spacetime fields $\phi$ unchanged, but transform the $X$ fields by $X \rightarrow X \circ Z$. This also transforms the pulled back fields $X^{*} \phi \rightarrow Z^{*} X^{*} \phi$, and due to the diffeomorphism invariance of the field equations, the pulled back fields still define solutions. These transformations therefore comprise a set of symmetries for the dynamics in the local subregion. Infinitesimally, these transformations are generated by vector fields $w^{a}$ on $\mathbb{R}^{d}$. Analogous to vector fields defined on $M, w^{a}$ defines a vector $\hat{w}$ on $\mathcal{S}$, whose action on the pulled back fields $X^{*} \phi$ is given by the Lie derivative,

$$
L_{\hat{w}} X^{*} \phi=£_{w} X^{*} \phi=X^{*} £_{\left(X^{-1}\right)^{*} w} \phi,
$$

while its action on $\phi$ is trivial, $L_{\hat{w}} \phi=0$. On the other hand, we may apply the pullback formula (3.1) to this equation to derive

$$
X^{*} £_{W} \phi=X^{*} I_{\hat{w}} £ \chi \phi,
$$

where $W^{a}=\left(X^{-1}\right)^{*} w^{a}$. The contractions of the vector $\hat{w}$ with the basic $\mathcal{S}$ one-forms are therefore

$$
I_{\hat{w}} \chi^{a}=W^{a}, \quad I_{\hat{w}} \delta \phi=0 .
$$

We also will assume that $w^{a}$ is independent of the solution, so that $\delta w^{a}=0$. Writing this as $0=\delta X^{*} W^{a}$, and applying the pullback formula (3.1), one finds

$$
\delta W^{a}=-£ \chi W^{a} .
$$

In order for the transformation to be a symmetry of the phase space, it must generate a Hamiltonian flow. This means that $I_{\hat{w}} \Omega$ is exact, and determines the Hamiltonian $H_{\hat{w}}$ for the flow via $\delta H_{\hat{w}}=-I_{\hat{w}} \Omega$. The contraction with the symplectic form can be computed straightforwardly from (4.12) by first using the decomposition (4.14) for $\delta Q \chi$. Then

$$
\begin{aligned}
I_{\hat{w}} \Omega & =\int_{\partial \Sigma}\left(-\Phi_{W}-Q_{[W, \chi]}-£_{\chi} Q_{W}+£_{W} Q_{\chi}+i_{W} \theta+i_{W} i_{\chi} L\right) \\
& =-\delta \int_{\partial \Sigma} Q_{W}+\int_{\partial \Sigma} i_{W}\left(\theta+I_{\hat{\chi}} \theta\right) .
\end{aligned}
$$

The first three terms of the first line combine into the first term in the second line, using formula (6.4) for $\delta W^{a}$, formula (4.14) for $\delta Q_{W}$, and recalling that the integral involves an implicit pullback by $X^{*}$, so that $\delta \int_{\partial \Sigma} Q_{W}=\int_{\partial \Sigma}\left(\delta Q_{W}+£_{\chi} Q_{W}\right)$. 
It is immediately apparent that if the second integral in (6.6) vanishes, the flow is Hamiltonian. This occurs if $W^{a}$ is tangent to $\partial \Sigma$ or vanishing at $\partial \Sigma$, and hence defines a mapping of the surface into itself. If $W^{a}$ is tangential, it generates a diffeomorphism $\partial \Sigma$, while vector fields that vanish on $\partial \Sigma$ generate transformations of the normal bundle to the surface while holding all points on the surface fixed. These transformations were respectively called surface diffeomorphisms and surface boosts in [36]. The remaining transformations consist of the surface translations, where $W^{a}$ has components normal to the surface, and the second integral in (6.6) does not vanish. In general, this term does not give a Hamiltonian flow, except when the fields satisfy certain boundary conditions. We will briefly discuss the surface translations in subsection 6.1 , where we show that they can give rise to central charges in the surface symmetry algebra.

Returning to the surface-preserving transformations, we find that the Hamiltonian is given by the Noether charge integrated over the boundary,

$$
H_{\hat{w}}=\int_{\partial \Sigma} Q_{W} .
$$

The surface symmetry algebra is generated through the Poisson bracket of the Hamiltonians for all possible surface-preserving vectors. The Poisson bracket is given by

$$
\left\{H_{\hat{w}}, H_{\hat{v}}\right\}=I_{\hat{w}} I_{\hat{v}} \Omega=-I_{\hat{w}} \delta \int_{\partial \Sigma} Q_{V}=-I_{\hat{w}} \int_{\partial \Sigma}\left(i_{V}+Q_{\delta V}+£_{\chi} Q_{V}\right)=\int_{\partial \Sigma} Q_{[W, V]},
$$

where the last equality uses equation (6.4) applied to $\delta V^{a}$ and that $\int_{\partial \Sigma} £_{W} Q_{V}=$ $\int_{\partial \Sigma} i_{W} d Q_{V}$ vanishes when integrated over the surface since $W^{a}$ is parallel to $\partial \Sigma$. This shows that the algebra generated by the Poisson bracket is compatible with the Lie algebra of surface preserving vector fields,

$$
\left\{H_{\hat{w}}, H_{\hat{v}}\right\}=H_{[w, v]},
$$

without the appearance of any central charges, i.e. the map $w^{a} \mapsto H_{\hat{w}}$ is a Lie algebra homomorphism. Note that the algebra of surface-preserving vector fields is much larger than the surface symmetry algebra. This is because the generators of surface symmetries depend only on the values of the vector field and its derivative at $\partial \Sigma$. Vector fields that die off sufficiently quickly near $\partial \Sigma$ correspond to vanishing Hamiltonians. The transformations they induce on $\mathcal{S}$ are pure gauge, and they drop out after passing to the reduced phase space.

To identify the surface symmetry algebra, it is useful to first describe the larger algebra of surface-preserving diffeomorphisms, which contains the surface symmetries as a subalgebra. It takes the form of a semidirect product, $\operatorname{Diff}(\partial \Sigma) \ltimes \operatorname{Dir}_{\partial \Sigma}$ where $\operatorname{Diff}(\partial \Sigma)$ is the diffeomorphism group of $\partial \Sigma$, and $\operatorname{Dir}_{\partial \Sigma}$ is the normal subgroup of diffeomorphisms that fix all points on $\partial \Sigma .^{9} \operatorname{Dir}_{\partial \Sigma}$ is generated by vector fields $W^{a}$ that vanish on $\partial \Sigma$, and it is a normal subgroup because the vanishing property is preserved under commutation with all surface-preserving vector fields:

$$
\left.[W, V]^{a}\right|_{\partial \Sigma}=\left.\left(W^{b} \partial_{b} V^{a}-V^{b} \partial_{b} W^{a}\right)\right|_{\partial \Sigma}=0,
$$

\footnotetext{
9 "Dir" stands for "Dirichlet," since these are the diffeomorphisms that would be consistent with fixed, Dirichlet boundary conditions at $\partial \Sigma$.
} 
where the first term vanishes since $W^{b}$ vanishes at $\partial \Sigma$, and the second term vanishes because $V^{b}$ is parallel to $\partial \Sigma$, and $W^{a}$ is zero everywhere along the surface. A general surface preserving vector field can then be expressed as

$$
W^{a}=W_{\|}^{a}+W_{0}^{a},
$$

where $W_{0}^{a}$ vanishes on $\partial \Sigma$ and $W_{\|}^{a}$ is tangent to $\partial \Sigma$. Note that this decomposition is not canonical; away from $\partial \Sigma$ there is some freedom in specifying which components of the vector field correspond to the tangential direction. However, given any such choice, it is clear that if $W_{\|}^{a}$ is nonvanishing at $\partial \Sigma$, then it will be nonzero in a neighborhood of $\partial \Sigma$, and hence the parallel vector fields act nontrivially on the $V_{0}^{a}$ component of other vector fields. Finally, the commutator of two purely parallel vector fields $\left[W_{\|}, V_{\|}\right]$will remain purely parallel, since they are tangent to an integral submanifold. The map $W^{a} \mapsto W_{\|}^{a}$ is therefore a homomorphism from the surface-preserving diffeomorphisms onto $\operatorname{Diff}(\partial \Sigma)$, with kernel Dir ${ }_{\partial \Sigma}$. This establishes that the group of surface-preserving diffeomorphisms is $\operatorname{Diff}(\partial \Sigma) \ltimes \operatorname{Dir}_{\partial \Sigma}$.

The surface symmetry algebra is represented as a subalgebra of $\operatorname{Diff}(\partial \Sigma) \ltimes \operatorname{Dir}_{\partial \Sigma}$. The Hamiltonian for a surface-preserving vector field is determined by the Noether charge $Q_{W}$, which depends only on the value of $W^{a}$ and its first derivative at $\partial \Sigma$. Hamiltonians for vector fields that are nonvanishing at $\partial \Sigma$ provide a faithful representation of the $\operatorname{Diff}(\partial \Sigma)$ algebra; however, the vanishing vector fields only represent a subalgebra of Dir $\partial \Sigma$. To determine it, note that only the first derivative of $W^{a}$ contributes to the Noether charge, and its tangential derivative vanishes. Letting $x^{i}, i=0,1$, represent coordinates in the normal directions that vanish on $\partial \Sigma$, the components of the vector field may be expressed $W^{\mu}=x^{i} W_{i}^{\mu}+\mathcal{O}\left(x^{2}\right), \mu=0, \ldots, d-1$, and the $\mathcal{O}\left(x^{2}\right)$ terms are determined by the second derivatives, which do not contribute to the Noether charge. Then the commutator of two vectors is

$$
[W, V]^{\mu}=x^{i}\left(W_{i}^{j} V_{j}{ }^{\mu}-V_{i}{ }^{j} W_{j}{ }^{\mu}\right)+\mathcal{O}\left(x^{2}\right),
$$

which is seen to be determined by the matrix commutator of $W_{i}{ }^{\mu}$ and $V_{j}{ }^{\nu}$, by allowing the $i, j$ indices to run over $0, \ldots, d-1$, setting all entries with $i, j>1$ to zero.

This algebra gives a copy of $\operatorname{SL}(2, \mathbb{R}) \ltimes \mathbb{R}^{2 \cdot(d-2)}$ for each point on $\partial \Sigma$. The abelian normal subgroup $\mathbb{R}^{2 \cdot(d-2)}$ is generated by vectors for which the $\mu$ index in $W_{i}{ }^{\mu}$ is tangential, i.e. $W_{i}{ }^{j} \equiv W_{i}{ }^{\mu} \nabla_{\mu} x^{j}=0$. These vectors represent shearing transformations of the normal bundle: they generate flows that vanish on $\partial \Sigma$, and are parallel to $\partial \Sigma$ away from the surface. By specifying a normal direction, one obtains a homomorphism sending $W_{i}{ }^{\mu}$ to its purely normal part, $W_{i}{ }^{j}$. The fact that only the traceless part of $\nabla_{a} W^{b}$ contributes to the Noether charge, which follows from the antisymmetry of $E^{a b c d}$ from equation (4.13) in $c$ and $d$, translates to the requirement that $W_{i}{ }^{j}$ be traceless when $W^{a}$ vanishes on $\partial \Sigma$. This means that the $2 \times 2$ matrices $W_{i}^{j}$ generate an $\operatorname{SL}(2, \mathbb{R})$ algebra. The generators $V_{i}{ }^{\mu}$ of $\mathbb{R}^{2 \cdot(d-2)}$ transform as a collection of $(d-2)$ vectors under the $\operatorname{SL}(2, \mathbb{R})$ algebra, verifying the semidirect product structure $\mathrm{SL}(2, \mathbb{R}) \ltimes \mathbb{R}^{2 \cdot(d-2)}$ for the vector fields vanishing at $\partial \Sigma$. Under diffeomorphisms of $\partial \Sigma, V_{i}{ }^{\mu}$ transforms as a pair of vectors; hence, the full surface symmetry algebra is $\operatorname{Diff}(\partial \Sigma) \ltimes\left(\operatorname{SL}(2, \mathbb{R}) \ltimes \mathbb{R}^{2 \cdot(d-2)}\right)^{\partial \Sigma}$. 
The extra factor of $\mathbb{R}^{2 \cdot(d-2)}$ is a novel feature of this analysis, appearing for generic higher curvature theories, but not for general relativity [36]. Its presence or absence is explained by the particular structure of $E^{a b c d}$, the variation of the Lagrangian scalar with respect to $R_{a b c d}$. When $E^{a b c d}$ is determined by its trace, i.e., equal to $\frac{E}{d(d-1)}\left(g^{a c} g^{b d}-g^{a d} g^{b c}\right)$ with $E$ a scalar, the $\mathbb{R}^{2 \cdot(d-2)}$ transformations are pure gauge. The Noether charge for a vector field vanishing at the surface evaluates to ${ }^{10}$

$$
\left.Q_{W}\right|_{\partial \Sigma}=\mu n_{a b} E_{d}^{a b c} \nabla_{c} W^{d}=\mu \frac{E}{d(d-1)} n_{d}^{c} \nabla_{c} W^{d},
$$

where $\mu$ is the volume form on $\partial \Sigma$ and $n_{a b}$ is the binormal; $n_{d}^{c}$ projects out the tangential component in $\nabla_{c} W^{d}$, leaving only the $\mathrm{SL}(2, \mathbb{R})$ transformations as physical symmetries. A particular class of theories in which this occurs are $f(R)$ theories (which include general relativity), where the Lagrangian is a function of the Ricci scalar, and $E^{a b c d}=\frac{1}{2} f^{\prime}(R)\left(g^{a c} g^{b d}-g^{a d} g^{b c}\right)$. In more general theories, however, $n_{a b} E_{d}^{a b c}$ will have a tangential component on the $d$ index, and the algebra enlarges to include the $\mathbb{R}^{2 \cdot(d-2)}$ tranformations.

Curiously, there always exists a choice of ambiguity terms, discussed in subsection 5.2, that eliminates the $\mathbb{R}^{2 \cdot(d-2)}$ symmetries. Namely, the symplectic potential current $\theta$ can be modified as in equation (5.4a), with $\beta$ chosen to be

$$
\beta=\epsilon_{a b} E^{a b e d} s_{e}^{c} \delta g_{c d}
$$

and $s_{e}{ }^{c}=-u_{e} u^{c}+n_{e} n^{c}$ is the projector onto the normal bundle of $\partial \Sigma$. Note that the explicit use of normal vectors to $\partial \Sigma$ makes this $\beta$ not spacetime-covariant. This is nevertheless in line with the broader set of allowed ambiguity terms discussed above. From equation (5.4d), this term changes the Noether charge of a vector vanishing at $\partial \Sigma$ to

$$
\left.Q_{W}\right|_{\partial \Sigma}=\mu n_{a b}\left(E_{d}^{a b c}-E_{d}^{a b e} s_{e}^{c}-E^{a b e c} s_{e d}\right) \nabla_{c} W^{d} .
$$

The additional terms involving $s_{e}{ }^{c}$ drop out when contracted with the normal component on the $d$ index of $\nabla_{c} W^{d}$; however, on the tangential component the additional terms cancel against the first term. This choice of ambiguity thus reduces the surface symmetry algebra to coincide with the algebra for general relativity, $\operatorname{Diff}(\partial \Sigma) \ltimes \mathrm{SL}(2, \mathbb{R})^{\partial \Sigma}$.

Whether or not to use this choice of $\beta$ depends on the application at hand, and it is unclear at the moment how exactly $\beta$ should be fixed when trying to characterize the edge mode contribution to the entanglement entropy of a subregion. The above choice is natural in the sense that it gives the same surface symmetry algebra for any diffeomorphisminvariant theory. This would mean that the surface symmetry algebra is determined by the gauge group of the theory, while the Hamiltonians for the symmetry generators change depending on the specific dynamical theory under consideration. Note also that there are additional ambiguity terms that could be added, some of which enlarge the symmetry algebra by introducing dependence on higher derivatives of the vector field. Determining how to fix the ambiguity remains an important open problem for the extended phase space program.

\footnotetext{
${ }^{10}$ The binormal is defined to be $n_{a b}=2 u_{[a} n_{b]}$ where $u_{a}$ is the timelike unit normal and $n_{a}$ is the inwardpointing spacelike unit normal. The spacetime volume form at $\partial \Sigma$ is then $\left.\epsilon_{a b}\right|_{\partial \Sigma}=-n_{a b} \wedge \mu$.
} 


\subsection{Surface translations}

While the surface-preserving transformations are present for generic surfaces, in situations where the fields satisfy certain boundary conditions at $\partial \Sigma$, the surface-symmetry algebra can enhance to include surface translations. These are generated by vector fields that contain a normal component to $\partial \Sigma$ on the surface. For such a vector field, the second integral in (6.6) does not vanish, so for this transformation to be Hamiltonian, this integral must be an exact $\mathcal{S}$ form. To understand when this can occur, it is useful to first rewrite the integral in terms of pulled back fields on $\partial \sigma$, the preimage of $\partial \Sigma$ under the $X$ map:

$$
\int_{\partial \Sigma} i_{W}\left(\theta+I_{\hat{\chi}} \theta\right)=\int_{\partial \sigma} X^{*} i_{W} \theta[\phi ; \delta \phi+£ \chi \phi]=\int_{\partial \sigma} i_{w} \theta\left[X^{*} \phi ; \delta X^{*} \phi\right] .
$$

Since $\delta w^{a}=0$, it is clear from this last expression that the flow will be Hamiltonian only if at the boundary, $\theta$ is exact when contracted with $w^{a}$,

$$
\left.i_{w} \theta\left[X^{*} \phi ; \delta X^{*} \phi\right]\right|_{\partial \sigma}=i_{w} \delta X^{*} B
$$

where $B[\phi]$ is some functional of the fields, possibly involving structures defined only at $\partial \Sigma$ such as the extrinsic curvature. When this condition is satisfied, the second integral in (6.6) simply becomes $\delta \int_{\partial \Sigma} i_{W} B$, and so the full Hamiltonian for an arbitrary vector field $w^{a}$ is

$$
H_{\hat{w}}=\int_{\partial \Sigma}\left(Q_{W}-i_{W} B\right) .
$$

Next we compute the algebra of the surface symmetry generators under the Poisson bracket. It is worth noting first that by contracting equation (6.17) with $I_{\hat{v}}$, we find that the $B$ functional satisfies

$$
\left.i_{W} £_{V} B\right|_{\partial \Sigma}=i_{W} I_{\hat{V}} \theta=i_{W}\left(d Q_{V}+i_{V} L\right) .
$$

With this, the Poisson bracket is given by

$$
\begin{aligned}
\left\{H_{\hat{w}}, H_{\hat{v}}\right\} & =-I_{\hat{w}} \delta \int_{\partial \Sigma}\left(Q_{V}-i_{V} B\right) \\
& =\int_{\partial \Sigma}\left(-I_{\hat{w}} \delta Q_{V}-£_{W} Q_{V}+I_{\hat{w}} i_{\delta V} B+£_{W} i_{V} B\right) \\
& =\int_{\partial \Sigma}\left(Q_{[W, V]}-i_{[W, V]} B\right)+\int_{\partial \Sigma} i_{W}\left(-d Q_{V}+£_{V} B-i_{V} d B\right) \\
& =H_{[w, v]^{\wedge}}+\int_{\partial \Sigma} i_{W} i_{V}(L-d B) .
\end{aligned}
$$

Hence, the commutator algebra of the vector fields $w^{a}$ is represented by the algebra provided by the Poisson bracket, except when both vector fields have normal components at the surface, in which case the second term in (6.20) gives a modification. In fact, the quantities

$$
K[\hat{w}, \hat{v}] \equiv \int_{\partial \Sigma} i_{W} i_{V}(L-d B)
$$


provide a central extension of the algebra, which is verified by showing that they are locally constant on the phase space, and hence commute with all generators. The exterior derivative is

$$
\begin{aligned}
\delta K[\hat{w}, \hat{v}] & =\int_{\partial \Sigma}\left[\delta i_{W} i_{V}(L-d B)+£_{\chi} i_{W} i_{V}(L-d B)\right] \\
& =\int_{\partial \Sigma} i_{W} i_{V}(\delta L-d \delta B) .
\end{aligned}
$$

On shell, we have $\delta L=d \theta$, and from (6.17) we can argue that the replacement $i_{W} i_{V} d \delta B \rightarrow$ $i_{W} i_{V} d \theta$ is valid at $\partial \Sigma$. Hence, the above variation vanishes, and $K[\hat{w}, \hat{v}]$ indeed defines a central extension of the algebra.

The modification that $B$ makes to the symmetry generators takes the same form as a Noether charge ambiguity arising from changing the Lagrangian $L \rightarrow L+d \alpha$, with $\alpha=-B$. Using the modified Lagrangian $L-d B$, the potential current changes to $\theta-\delta B$. The boundary condition (6.17) then implies that the terms involving $\theta$ in (6.6) vanish. The symmetry generators are simply given by the integrated Noether charge, which is modified to $Q_{W} \rightarrow Q_{W}-i_{W} B$ by the ambiguity. Hence, the generators $H_{\hat{w}}$ are the same as in (6.18), and their Poisson brackets still involve the central charges $K[\hat{w}, \hat{v}]$. Finally, note that the constancy of the central charges requires the variation of the modified Lagrangian $L-d B$ be zero when evaluated on $\partial \Sigma$. Requiring that variations of the Lagrangian have no boundary term on shell generally determines the boundary conditions for the theory. The same is true here: a choice of $B$ satisfying (6.17) can generally only be found if the fields obey certain boundary conditions, and different boundary conditions lead to different choices for $B$.

The surface translations can be parameterized by normal vector fields $W^{i}$ defined on $\partial \Sigma$. Assuming $\partial_{i} W^{j}=0$ in some coordinate system, where $i, j$ are normal indices, we can work out their commutation relations with generators of the rest of the algebra:

$$
\begin{aligned}
{\left[W^{i}, V^{j}\right] } & =0 \\
{\left[W^{i}, x^{j} V_{j}{ }^{k}\right] } & =W^{i} V_{i}{ }^{k} \\
{\left[W^{i}, V^{A}\right] } & =-V^{A} \partial_{A} W^{i} \\
{\left[W^{i}, x^{j} V_{j}{ }^{A}\right] } & =W^{i} V_{i}{ }^{A}-x^{j} V_{j}{ }^{A} \partial_{A} W^{i},
\end{aligned}
$$

where $A$ denotes a tangential index. The first relation shows that the new generators commute among themselves (although the corresponding Poisson bracket is equal to the central charge $K[\hat{w}, \hat{v}])$, while the second and third show that $W^{i}$ transforms as a vector under $\operatorname{SL}(2, \mathbb{R})$ and as a scalar under $\operatorname{Diff}(\partial \Sigma)$. If the Noether charge ambiguity is chosen as in equation (6.14) so that the normal shearing generators $x^{j} V_{j}{ }^{A}$ drop out of the algebra, the resulting surface symmetry algebra is $\operatorname{Diff}(\partial \Sigma) \ltimes\left(\operatorname{SL}(2, \mathbb{R}) \ltimes \mathbb{R}^{2}\right)^{\partial \Sigma}$. However, if the normal shearing transformations are retained, equation (6.26) shows that the surface translations are no longer a normal subgroup, since the commutator gives rise to generators of $\operatorname{Diff}(\partial \Sigma)$ and $\operatorname{SL}(2, \mathbb{R})^{\partial \Sigma}$. In this case, the full surface symmetry algebra is simple.

The above analysis was carried out assuming that all normal vectors generate a surface symmetry. In practice, equation (6.17) may only be obeyed for some specifically chosen 
normal vectors [44]. The resulting algebra will then be a subalgebra of the generic case considered in this section.

\section{Discussion}

Building on the results of [36], this paper has described a general procedure for constructing the extended phase space in a diffeomorphism-invariant theory for a local subregion. The integral of the symplectic current for the unextended theory fails to be degenerate for diffeomorphisms that act at the boundary, and this necessitates the introduction of new fields, $X$, to ensure degeneracy. These fields can be thought of as defining a coordinate system for the local subregion, and the extended solution space consists of fields satisfying the equations of motion in all possible coordinate systems parameterized by $X$. While the $X$ fields do not satisfy dynamical equations themselves, it was shown in section 4 that their variations contribute to the symplectic form through the boundary integral in equation (4.12).

There are a few novel features of the extended phase space for arbitrary diffeomorphism-invariant theories that do not arise in vacuum general relativity with zero cosmological constant. First, in any theory whose Lagrangian does not vanish on-shell, the symplectic potential $\Theta$ is not a single-valued one form on the reduced phase space $\mathcal{P}$. This is due to the bulk integral of the Lagrangian that appears in equation (4.9), along with the fact that variations for which $\chi^{a}$ has support only away from the boundary $\partial \Sigma$ are degenerate directions of the extended symplectic form, (4.12). Because of this, $\Omega$ fails to be exact, despite satisfying $\delta \Omega=0$. Investigating the consequences of this nontrivial cohomology for $\mathcal{P}$ remains an interesting topic for future work.

Another new result comes from the form of the surface symmetry algebra. As in general relativity, any phase space transformation generated by $\hat{w}$ for which $W^{a} \equiv I_{\hat{w}} \chi^{a}$ is tangential at $\partial \Sigma$ is Hamiltonian. These generate the group $\operatorname{Diff}(\partial \Sigma) \ltimes \operatorname{Dir}_{\partial \Sigma}$ of surfacepreserving diffeomorphisms, but only a subgroup is represented on the phase space. This subgroup was found in section 6 to be $\operatorname{Diff}(\partial \Sigma) \ltimes\left(\operatorname{SL}(2, \mathbb{R}) \ltimes \mathbb{R}^{2 \cdot(d-2)}\right)^{\partial \Sigma}$, which is larger than the surface symmetry group $\operatorname{Diff}(\partial \Sigma) \ltimes \mathrm{SL}(2, \mathbb{R})^{\partial \Sigma}$ found in [36] for general relativity. The additional abelian factor $\mathbb{R}^{2 \cdot(d-2)}$ arises generically; however, it is not present in $f(R)$ theories, in which the tensor $E^{a b c d}$ is constructed solely from the metric and scalars. We also noted that for any theory, there exists a choice (6.14) of ambiguity terms that can be added to $\theta$, with the effect of eliminating the $\mathbb{R}^{2 \cdot(d-2)}$ factor of the surface symmetry algebra.

The inclusion of surface translations into the surface symmetry algebra was discussed in section 6.1. This requires the existence of a $(d-1)$-form $B$ satisfying the relation (6.17) for at least some vector fields that are normal to the boundary. If such a form can be found, the surface translations are generated by the Hamiltonians (6.18). Interestingly, the Poisson brackets of these Hamiltonians acquire central charges given by (6.21), which depend on the on-shell value of the modified Lagrangian $L-d B$ at $\partial \Sigma$. Such central charges are a common occurrence in surface symmetry algebras that include surface translations [44, 45, 49, 64-67]. In general, the existence of $B$ requires that the fields satisfy boundary conditions at $\partial \Sigma$. An important topic for future work would be to classify which 
boundary conditions the fields must satisfy in order for $B$ to exist. For example, with Dirichlet boundary conditions where the field values are specified at $\partial \Sigma, B$ is given by the Gibbons-Hawking boundary term, constructed from the trace of the extrinsic curvature in the normal direction [68]. However, such boundary conditions are quite restrictive on the dynamics. For a local subsystem in which $\partial \Sigma$ simply represents a partition of a spatial slice, one would not expect Dirichlet conditions to be compatible with all solutions of the theory. An alternative approach would be to impose conditions that specify the location of the surface in a diffeomorphism-invariant manner, without placing any restriction on the dynamics. One example is requiring that the surface extremize its area or some other entropy functional, as is common in holographic entropy calculations [2, 59-61, 69, 70]. Since extremal surfaces exist in generic solutions, these boundary conditions put no dynamical restrictions on the theory, but rather restrict where the surface $\partial \Sigma$ lies.

The effects of JKM ambiguity terms in the extended phase space construction were discussed in section 5 . It was noted that the $B$ form that appears when analyzing the surface translations could be interpreted as a Lagrangian ambiguity, $L \rightarrow L-d B$. Note that this type of ambiguity does not affect the symplectic form (4.12), and, as a consequence, the generators of the surface symmetries do not depend on this replacement. In fact, the generators (6.18) are invariant with respect to additional changes to the Lagrangian $L \rightarrow L+d \alpha$, since such a change shifts the Noether charge $Q_{W} \rightarrow Q_{W}+i_{W} \alpha$, but also induces the change $B \rightarrow B+\alpha$. An ambiguity that does affect the phase space is the shift freedom in the symplectic potential current, $\theta \rightarrow \theta+d \beta$. We noted that certain choices of $\beta$ can change the number of edge mode degrees of freedom, and also can affect the surface symmetry algebra. In the future, we would like to understand how this ambiguity should be fixed. One idea would be to use the ambiguity to ensure some $B$ can be found satisfying equation (6.17). In this case, the ambiguity is fixed as an integrability condition for $\theta$. Such an approach seems related to the ideas of [62] in which the ambiguity was chosen to give an entropy functional satisfying a linearized second law. Another approach discussed in [61, 70-72] fixes the ambiguity through the choice of metric splittings that arise when performing the replica trick in the computation of holographic entanglement entropy.

As discussed in the introduction, one of the main motivations for constructing the extended phase space is to understand entanglement entropy in diffeomorphism-invariant theories [36]. The Hilbert space for such a theory does not factorize across an entangling surface due to the constraints. However, one can instead construct an extended Hilbert space for a local subregion as a quantization of the extended phase space constructed above. This extended Hilbert space will contain edge mode degrees of freedom that transform in representations of the surface symmetry algebra. A similar extended Hilbert space can be constructed for the complementary region with Cauchy surface $\bar{\Sigma}$, whose edge modes and surface symmetries will match those associated with $\Sigma$. The physical Hilbert space for $\Sigma \cup \bar{\Sigma}$ is given by the so-called entangling product of the two extended Hilbert spaces, which is the tensor product modded out by the action of the surface symmetry algebra. One then finds that the density matrix associated with $\Sigma$ splits into a sum over superselection sectors, labelled by the representations of the surface symmetry group. 
This block diagonal form of the density matrix leads to a von Neumann entropy that is the sum of three types of terms,

$$
S=\sum_{i}\left(p_{i} S_{i}-p_{i} \log p_{i}+p_{i} \log \operatorname{dim} R_{i}\right)
$$

where the sum is over the representations $R_{i}$ of the surface symmetry group, $p_{i}$ give the probability of being in a given representation, and $S_{i}$ is the von Neumann entropy within each superselection sector. The first term represents the average entropy of the interior degrees of freedom, while the second term is a classical Shannon entropy coming from uncertainty in the surface symmetry representation corresponding to the state. The last term arises from entanglement between the edge modes themselves, and is only present for a nonabelian surface symmetry algebra [73, 74]. The dimension of the representation has some expression in terms of the Casimirs of the group, and hence this term will take the form of an expectation value of local operators at the entangling surface. It is conjectured that this term provides a statistical interpretation for the Wald-like contributions in the generalized entropy, $S_{\text {gen }}=S_{\text {Wald-like }}+S_{\text {out }}$ [36]. Put another way, given a UV completion for the quantum gravitational theory, the edge modes keep track of the entanglement between the UV modes that are in a fixed state, corresponding to the low energy "code subspace" $[8,75]$.

On reason for considering the extended phase space in the context of entanglement entropy comes from issues of divergences in entanglement entropy. These divergences arise generically in quantum field theories, and a regulation prescription is needed in order to get a finite result. A common regulator for Yang-Mills theories is a lattice [1, 73, 74], which preserves the gauge invariance of the theory. Unfortunately, a lattice breaks diffeomorphism invariance, which can be problematic when using it as a regulator for gravitational theories (see [76] for a review of the lattice approach to quantum gravity). The extended phase space provides a continuum description of the edge modes that respects diffeomorphism invariance. As such, it should be amenable to finding a regulation prescription that does not spoil the gauge invariance of the gravitational theory. Finding such a description is an important next step in defining entanglement entropy for a gravitational theory.

There are a number of directions for future work on the extended phase space itself, outside of its application to entanglement entropy. One topic of interest is to clarify the fiber bundle geometry of the solution space $\mathcal{S}$, which arises due to diffeomorphism invariance. A fiber in this space consists of all solutions that are related by diffeomorphism, and the $\chi^{a}$ fields define a flat connection on the bundle. Flatness in this case is equivalent to the equation $\delta\left(\chi^{a}\right)+\frac{1}{2}[\chi, \chi]^{a}=0$ for the variation of $\chi^{a}$. This fiber bundle description of $\mathcal{S}$ will be reported on in a future work [77]. Another technical question that arises is whether $\mathcal{S}$ truly carries a smooth manifold structure. One obstruction to smoothness would be if the equations of motion are not well-posed in some coordinate system. In this case, the solutions do not depend smoothly on the initial conditions on the Cauchy slice $\Sigma$, calling into question the smooth manifold structure of $\mathcal{S}$. If $X$ is used to define the coordinate system, this would mean that for some values of $X$ the solution space is not smooth. A possible way around this is to always work in a coordinate system in which the field equations are well-posed, and the gauge transformation to this coordinate system would 
impose dynamical equations on the $X$ fields. Another obstruction to smoothness comes from issues related to ergodicity and chaos in totally constrained systems [78]. It would be interesting to understand if these issues are problematic for the phase space construction given here, and whether the $X$ fields ameliorate any of these problems.

Another interesting application would be to formulate the first law of black hole mechanics and various related ideas in terms of the extended phase space. This could be particularly interesting in clarifying certain gauge dependence that appears when looking at second order perturbative identities, such as described in [79]. The edge modes should characterize all possible gauge choices, and they may inform some of the relations found in $[16,80,81]$ when considering different gauges besides the Gaussian null coordinates used in [79]. They could also be useful in understanding quasilocal gravitational energy, and in particular how to define the gravitational energy inside a small ball. This can generally be determined by integrating a pseudotensor over the ball, but there is no preferred choice for a gravitational pseudotensor, so this procedure is ambiguous. It would be interesting if a preferred choice presented itself by considering second order variations of the first law of causal diamonds $[17,20]$, using the extended phase space. Some ideas in this direction are being considered in [82], but it is difficult to find a quasilocal gravitational energy that satisfies the desirable property of being proportional to the Bel-Robinson energy density in the small ball limit $[83,84]$.

Finally, it would be very useful to recast the extended phase space construction in vielbein variables. Some progress on the vielbein formulation was reported in [48]. Since vielbeins have an additional internal gauge symmetry associated with local Lorentz invariance, care must be taken when applying covariant canonical constructions $[85,86]$. It would be particularly interesting to analyze the surface symmetry algebra that arises in this case, which could differ from the algebra derived using metric variables because the gauge group is different. Comparing the algebras and edge modes in both cases would weigh on the question of how physically relevant and universal their contribution to entanglement entropy is.

\section{Acknowledgments}

I would like to thank William Donnelly, Laurent Freidel, Ted Jacobson, Eric Mintun and Arif Mohd for helpful discussions, and Daniel Brennan for comments on a draft of this work. I thank the organizers of the TASI 2017 Summer School, where a portion of this work was completed. This research is supported by the National Science Foundation under grant No. PHY-1407744.

\section{A List of identities}

This appendix gives a collection of identities for the exterior calculus on solution space $\mathcal{S}$ along with their proofs.

A.1 $L_{V}=I_{V} \delta+\delta I_{V}$

Proof. This follows from standard treatments of the exterior calculus [51]. 
A.2 $L_{V} I_{U}=I_{[V, U]}+I_{U} L_{V}$

Proof. This is simply the derivation property of the Lie derivative applied to all tensor fields on $\mathcal{S}$. $I_{U} \alpha$ is a contraction of the vector $U$ with the one-form $\alpha$, so the Lie derivative first acts on $U$ to give the vector field commutator $L_{V} U=[V, U]$, and then acts on $\alpha$, with the contraction $I_{U}$ now being applied to $L_{V} \alpha$. Hence, on an arbitrary form, $L_{V} I_{U} \alpha=I_{[V, U]} \alpha+I_{U} L_{V} \alpha$.

A.3 $L_{V} Y^{*} \alpha=Y^{*}\left(L_{V} \alpha+£_{\left(I_{V} \chi_{Y}\right)} \alpha\right)$

Proof. The discussion of section 2 derived equation (2.4), so all that remains is to show that $\chi^{a}(Y ; V)$ is linear in the vector $V$. This can be demonstrated inductively on the degree of $\alpha$. For scalars, it is enough to show it holds on the functions $\phi^{x}$. Applying A.1, we have on the one hand

$$
L_{V} Y^{*} \phi=I_{V} \delta Y^{*} \phi,
$$

while on the other hand,

$$
L_{V} Y^{*} \phi=Y^{*}\left(L_{V} \phi+£_{\chi(Y ; V)} \phi\right)=I_{V} Y^{*} \delta \phi+Y^{*} £_{\chi(Y ; V)} \phi
$$

since $I_{V}$ commutes with $Y^{*}$. Equating these expressions, we find

$$
Y^{*} \chi_{\chi(Y ; V)} \phi=I_{V}\left(\delta Y^{*} \phi-Y^{*} \delta \phi\right) .
$$

Since the right hand side of this expression is linear in $V, \chi(Y ; V)$ must be as well.

Now suppose A.3 holds for all forms of degree $n-1$, and take $\alpha$ to be degree $n$. Then for an arbitrary vector $U, I_{U} Y^{*} \alpha$ is degree $n-1$, so

$$
L_{V} I_{U} Y^{*} \alpha=Y^{*}\left(L_{V} I_{U} \alpha+£_{\left(I_{V} \chi_{Y}\right)} I_{U} \alpha\right)=I_{[V, U]} Y^{*} \alpha+I_{U} Y^{*}\left(L_{V} \alpha+£_{\left(I_{V} \chi_{Y}\right)} \alpha\right),
$$

where identity A.2 was applied along with the fact that $I_{U}$ commutes with $£_{\xi}$. On the other hand,

$$
L_{V} I_{U} Y^{*} \alpha=I_{[V, U]} Y^{*} \alpha+I_{U} L_{V} Y^{*} \alpha=I_{[V, U]} Y^{*} \alpha+I_{U} Y^{*}\left(L_{V} \alpha+I_{\bar{\chi}(Y ; V)} \alpha\right) .
$$

Since $U$ was arbitrary, equating these expressions shows that $\bar{\chi}^{a}(Y ; V)=I_{V} \chi_{Y}^{a}$, showing that the formula holds for forms of degree $n$.

A.4 $I_{V} £_{\chi_{Y}}=£_{\left(I_{V} \chi_{Y}\right)}-£_{\chi_{Y}} I_{V}$

Proof. This is essentially the antiderivation property applied to $£ \chi_{Y}$. The spacetime Lie derivative $£ \chi_{Y}$ acting on a tensor can be written in terms of $\chi_{Y}^{a}$ and its derivatives contracted with the tensor, where all instances of $\chi_{Y}^{a}$ appear to the left. It is straightforward to see that when $I_{V}$ contracts with $\chi_{Y}^{a}$ in this expression, the terms will combine into $£_{\left(I_{V} \chi_{Y}\right)}$, and since $I_{V}$ does not change the spacetime tensor structure of the object it contracts, the remaining terms will combine into $-£ \chi_{Y} I_{V}$, with the minus coming from the antiderivation property of $I_{V}$. 
A.5 $\delta Y^{*} \alpha=Y^{*}\left(\delta \alpha+£ \chi_{Y} \alpha\right)$

Proof. This may also be demonstrated inductively on the degree of $\alpha$. For scalars, we simply note that equation (A.3) is valid for arbitrary vectors $V$, and since $\chi^{a}(Y ; V)=$ $I_{V} \chi_{Y}^{a}$, we derive $\delta Y^{*} \phi=Y^{*}\left(\delta \phi+£ \chi_{Y} \phi\right)$. Assume now A.5 holds for all $(n-1)$-forms, and take $\alpha$ an $n$-form and $V$ an arbitrary vector. Then

$$
\begin{aligned}
I_{V} \delta Y^{*} \alpha & =L_{V} Y^{*} \alpha-\delta I_{V} Y^{*} \alpha \\
& =Y^{*}\left(L_{V} \alpha+£_{\left(I_{V} \chi_{Y}\right)} \alpha-\delta I_{V} \alpha-£ \chi_{Y} I_{V} \alpha\right) \\
& =I_{V} Y^{*}\left(\delta \alpha+£ \chi_{Y} \alpha\right)
\end{aligned}
$$

The first equality applies A.1, the second uses A.3 and the fact that $I_{V} Y^{*} \alpha$ is an $(n-1)$-form, and the last equality follows from A.1 and A.4. Since $V$ is arbitrary, this completes the proof.

A.6 $\frac{1}{2}\left[\chi_{Y}, \chi_{Y}\right]^{a}=\chi_{Y}^{b} \nabla_{b} \chi_{Y}^{a}$

Proof. This is a consequence of the formula for the commutator of two vectors, $[\xi, \zeta]=$ $\xi^{b} \nabla_{b} \zeta^{a}-\zeta^{b} \nabla_{b} \xi^{a}$, along with the fact that since $\chi^{a}$ is an $\mathcal{S}$ one-form, it anticommutes with itself. Alternatively, the formula may be checked by contracting with arbitrary vectors $V$ and $U$. Letting $I_{V} \chi_{Y}^{a}=-\xi^{a}$ and $I_{U} \chi_{Y}^{a}=-\zeta^{a}$, we have

$$
I_{V} I_{U} \frac{1}{2}\left[\chi_{Y}, \chi_{Y}\right]^{a}=I_{V}\left[\chi_{Y}, \zeta\right]^{a}=[\zeta, \xi]^{a}=\zeta^{b} \nabla_{b} \xi^{a}-\xi^{b} \nabla_{b} \zeta^{a}=I_{V} I_{U} \chi_{Y}^{b} \nabla_{b} \chi_{Y}^{a}
$$

A.7 $£_{\chi_{Y}} £_{\chi_{Y}}=£_{\frac{1}{2}\left[\chi_{Y}, \chi_{Y}\right]}$

Proof. For ordinary spacetime vectors $\xi^{a}$ and $\zeta^{a}$, the Lie derivative satisfies [58]

$$
£_{\xi} £_{\zeta}=£_{[\xi, \zeta]}+£_{\zeta} £_{\xi}
$$

Since $\chi_{Y}^{a}$ are anticommuting, this formula is modified to

$$
£_{\chi_{Y}} £_{\chi_{Y}}=£_{\left[\chi_{Y}, \chi_{Y}\right]}-£_{\chi_{Y}} £_{\chi_{Y}},
$$

from which the identity follows. Note that A.6 provides a formula for $\left[\chi_{Y}, \chi_{Y}\right]^{a}$.

A. $8 £_{\xi}\left(Y^{-1}\right)^{*}=\left(Y^{-1}\right)^{*} £_{Y^{*} \xi}$

Proof. This identity is a standard property of the Lie derivative, see e.g. [87].

A.9 $£_{\chi} i_{\chi}=\frac{1}{2}\left(i_{[\chi, \chi]}+d i_{\chi} i_{\chi}-i_{\chi} i_{\chi} d\right)$ 
Proof. The identity for ordinary spacetime vectors $\xi^{a}$ and $\zeta^{b}[58]$

$$
£_{\xi} i_{\zeta}=i_{[\xi, \zeta]}+i_{\zeta} £_{\xi}
$$

along with the fact that $\chi^{a}$ are anticommuting gives

$$
\begin{aligned}
£_{\chi} i_{\chi} & =i_{[\chi, \chi]}-i_{\chi} £_{\chi} \\
& =i_{[\chi, \chi]}-i_{\chi} d i_{\chi}-i_{\chi} i_{\chi} d \\
& =i_{[\chi, \chi]}-£_{\chi} i_{\chi}+d i_{\chi} i_{\chi}-i_{\chi} i_{\chi} d,
\end{aligned}
$$

and moving $-£ \chi i \chi$ to the left hand side proves the identity.

A.10 $£ \chi \theta+\delta i_{\chi} L+£ \chi i_{\chi} L=d\left(i \chi \theta+\frac{1}{2} i_{\chi} i_{\chi} L\right)$

Proof. The first term in this expression is $£ \chi \theta=d i \chi \theta+i \chi d \theta$, which gives one of the terms on the right hand side of the identity, along with $i_{\chi} d \theta$. Next we have

$$
\delta i_{\chi} L=i_{\delta \chi} L-i_{\chi} \delta L=-\frac{1}{2} i_{[\chi, \chi]} L-i_{\chi} d \theta,
$$

where we applied equation (2.8) for $\delta \chi^{a}$, and used that $\delta L=d \theta$ on shell. The $-i \chi d \theta$ term cancels against the similar term appearing in $£ \chi \theta$, so that the remaining pieces are

$$
-\frac{1}{2} i_{[\chi, \chi]} L+£ \chi i_{\chi} L=\frac{1}{2} d i_{\chi} i_{\chi} L,
$$

which follows from identity A.9 and $d L=0$. Hence, the terms on the left of the A.10 combine into the exact form $d\left(i_{\chi} \theta+\frac{1}{2} i_{\chi} i_{\chi} L\right)$.

$\mathrm{A} .11[V, \hat{\xi}]=\left(I_{V} \delta \xi^{a}\right)^{\wedge}$

Proof. Here we can use that on local $\mathcal{S}$-scalars, $L_{\hat{\xi}} \phi=£_{\xi} \phi$. Then

$$
L_{[V, \hat{\xi}]} \phi=L_{V} L_{\hat{\xi}} \phi-L_{\hat{\xi}} L_{V} \phi=L_{V} £_{\xi} \phi-£_{\xi} I_{V} \delta \phi=£_{\left(I_{V} \delta \xi\right)^{\wedge}} \phi=L_{\left(I_{V} \delta \xi\right)^{\wedge}} \phi,
$$

hence, $[V, \hat{\xi}]=\left(I_{V} \delta \xi^{a}\right)$.

A.12 $L_{\hat{\xi}}=£_{\xi}+I_{\delta \xi^{\wedge}}$

Proof. This formula is meant to apply to local functionals of the fields defined at a single spacetime point. Since $I_{\delta \xi^{\wedge}}$ annihilates scalars, it clearly is true for that case. Then assume the formula has been shown for all $(n-1)$-forms, and take $\alpha$ to be an $n$-form. For an arbitrary vector $V$, since $I_{V} \alpha$ is an $(n-1)$-form, we have

$$
\begin{aligned}
I_{V} L_{\hat{\xi}} \alpha & =L_{\hat{\xi}} I_{V} \alpha-I_{[\hat{\xi}, V]} \alpha=£_{\xi} I_{V} \alpha+I_{\delta \xi^{\prime}} I_{V} \alpha-I_{[\hat{\xi}, V]} \alpha \\
& =I_{V}\left(£_{\xi} \alpha+I_{\delta \xi^{\wedge}} \alpha\right)-I_{\left(I_{V} \delta \xi\right)^{\wedge}} \alpha-I_{[\hat{\xi}, V]} \alpha,
\end{aligned}
$$

and the last two terms in this expression cancel due to identity A.11. Since $V$ was arbitrary, we conclude that the identity holds for all $n$ forms, and by induction for all $\mathcal{S}$ differential forms. 
A.13 $L_{\hat{\chi}}=I_{\hat{\chi}} \delta-\delta I_{\hat{\chi}}$

Proof. This is essentially a definition of what is meant by $L_{\hat{\chi}}$. The left hand side is the graded commutator of the derivation $I_{\hat{\chi}}$ and the antiderivation $\delta$, which defines the antiderivation $L_{\hat{\chi}}[87]$.

A.14 $[V, \hat{\chi}]=\left(\delta I_{V} \chi^{a}\right)^{\wedge}-\left[I_{V} \chi, \chi\right]^{\wedge}$

Proof. This follows from the defining relation of the bracket [87],

$$
L_{V} L_{\hat{\chi}}-L_{\hat{\chi}} L_{V}=L_{[V, \hat{\chi}]} .
$$

Applied to $\phi$ and defining $\nu^{a}=-I_{V} \chi^{a}$, this gives

$$
\begin{aligned}
L_{[V, \hat{\chi}]} \phi & =\left(L_{V} L_{\hat{\chi}}-L_{\hat{\chi}} L_{V}\right) \phi \\
& =I_{V} \delta £_{\chi} \phi-\delta £_{\nu} \phi-£_{\chi} I_{V} \delta \phi \\
& =I_{V}\left(£_{\delta \chi} \phi-£_{\chi} \delta \phi\right)-£_{\delta \nu} \phi-£_{\nu} \delta \phi+I_{V} £_{\chi} \delta \phi \\
& =\left(£_{[\nu, \chi]}-£_{\delta \nu}\right) \phi \\
& =\left(L_{[\nu, \chi]^{\wedge}}-L_{\delta \nu^{\wedge}}\right) \phi,
\end{aligned}
$$

To get to the third line, the expression (2.8) for $\delta \chi^{a}$ was used. We then conclude $[V, \hat{\chi}]=[\nu, \chi]^{\wedge}-\delta \nu^{\wedge}$, proving the identity.

A.15 $L_{\hat{\chi}}=£_{\chi}-I_{\delta \chi}$

Proof. The formalism of graded commutators developed in [87] is a useful tool in proving this identity. Given two graded derivations $D_{1}$ and $D_{2}$, their graded commutator $D_{1} D_{2}-(-1)^{k_{1} k_{2}} D_{2} D_{1}$ is another graded derivation, where $k_{i}$ are the degrees of the respective derivations, i.e. the amount the derivation increases or decreases the degree of the form on which it acts. Hence, since $I_{V}$ and $L_{\hat{\chi}}$ are derivations of degrees -1 and 1 , they satisfy

$$
I_{V} L_{\hat{\chi}}+L_{\hat{\chi}} I_{V}=-L_{\hat{\nu}}+I_{[\hat{\chi}, V]},
$$

where $-\nu^{a}=I_{V} \chi^{a}$. Similarly, we have

$$
I_{V} I_{\delta \chi^{\wedge}}+I_{\delta \chi^{\wedge}} I_{V}=I_{\left(I_{V} \delta \chi\right)^{\wedge}}=I_{[\nu, \chi]^{\wedge}},
$$

where equation (2.8) was used in the last equality.

We then prove the identity through induction on the degree of the form on which it acts. It is true for scalars because $I_{\delta \chi} \phi=0$. Then suppose it is true for all $(n-1)$-forms, and take $\alpha$ to be an $n$-form. For an arbitrary vector $V$ we have

$$
\begin{aligned}
I_{V} L_{\chi} \alpha & =I_{[\hat{\chi}, V]} \alpha-L_{\hat{\nu}} \alpha-L_{\chi} I_{V} \alpha \\
& =I_{\delta \nu^{\wedge}} \alpha-I_{[\nu, \chi]^{\wedge}} \alpha-£_{\nu} \alpha-I_{\delta \nu^{\wedge}} \alpha-£_{\chi} I_{V} \alpha+I_{\delta \chi} I_{V} \alpha \\
& =I_{V}\left(£_{\chi} \alpha-I_{\delta \chi^{\wedge}} \alpha\right) .
\end{aligned}
$$


The first line employs equation (A.18), the second line uses identities A.14 and A.12 as well as the fact that $I_{V} \alpha$ is an $(n-1)$-form, and the third line employs equation (A.19). Since $V$ is arbitrary, we conclude the identity holds for all $n$-forms, which completes the proof.

\section{B Edge mode derivatives in the symplectic form}

In this appendix, we derive the result advertised in section 4, that the symplectic form (4.12) does not depend on second or higher derivatives of $\chi^{a}$. Derivatives of $\chi^{a}$ appear in $\Omega$ through the terms $\delta Q_{\chi}+£_{\chi} Q_{\chi}$. The Lie derivative term may be expressed

$$
\begin{aligned}
& £_{\chi} Q_{\chi}=L_{\hat{\chi}} Q_{\chi}+I_{\delta(\chi)^{\wedge}} Q_{\chi} \\
& =I_{\hat{\chi}} \delta Q_{\chi}-\delta I_{\hat{\chi}} Q_{\chi}-Q_{\delta(\chi)} \\
& =I_{\hat{\chi}}{ }^{\gamma} \chi+I_{\hat{\chi}} Q_{\delta(\chi)}+\delta Q_{\chi}-Q_{\delta(\chi)} \\
& =\varphi_{\chi}+I_{\hat{\chi}} \vartheta_{\chi}+Q_{[\chi, \chi]} \text {. }
\end{aligned}
$$

These steps invoke the identities A.15, A.13 and equations (2.8) and (3.5), as well as the defining relation (4.14) for $\varnothing$. Adding $\delta Q_{\chi}=\S_{\chi}-\frac{1}{2} Q_{[\chi, \chi]}$ to this yields

$$
\delta Q_{\chi}+£_{\chi} Q_{\chi}=2 \AA_{\chi}+I_{\chi} \AA_{\chi}+\frac{1}{2} Q_{[\chi, \chi]} .
$$

From the derivation property of $I_{\hat{\chi}}$ acting on $\mathcal{S}$-forms and the identity $I_{\hat{\chi}} \chi^{a}=-\chi^{a}$, it

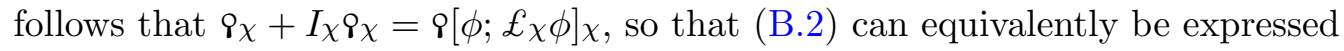

$$
\delta Q_{\chi}+£ \chi Q_{\chi}=9[\phi ; \delta \phi]_{\chi}+\varphi[\phi ; £ \chi \phi]_{\chi}+\frac{1}{2} Q_{[\chi, \chi]} .
$$

This expression is now amenable to determining how the derivatives of $\chi^{a}$ appear. Both $९\left[\phi ; £_{\chi} \phi\right]_{\chi}$ and $Q_{[\chi, \chi]}$ contain second derivatives. The relevant term in $९\left[\phi ; £_{\chi} \phi\right] \chi$ comes from the variation of the Christoffel symbol in (4.15), which gives

$$
\begin{aligned}
-\epsilon_{a b} E^{a b c d}\left(\nabla_{c} \nabla_{(d} \chi_{e)}+\nabla_{e} \nabla_{(d} \chi_{c)}-\nabla_{d} \nabla_{(c} \chi_{e)}\right) \chi^{e} \\
=-\frac{1}{2} \epsilon_{a b} E^{a b c d}\left(\nabla_{c} \nabla_{e} \chi_{d}-\nabla_{d} \nabla_{e} \chi_{c}\right) \chi^{e}+\text { n.d. } \\
=-\epsilon_{a b} E^{a b c}\left(\nabla_{(c} \nabla_{e)} \chi^{d}\right) \chi^{e}+\text { n.d. },
\end{aligned}
$$

where "n.d." represents terms with no derivatives acting on $\chi^{a}$. This derivation invokes the antisymmetry of $E^{a b c d}$ on $c$ and $d$, and collects all terms involving antisymmetrized derivatives of $\chi_{d}$ into the n.d. piece, since these can be replaced by a Riemann tensor contracted with an undifferentiated $\chi_{d}$.

Second derivatives of $\chi^{d}$ also appear in $\frac{1}{2} Q_{[\chi, \chi]}$ through the $E_{d}^{a b c}$ term in the equation (4.13) for the Noether charge. This term evaluates to

$$
\begin{aligned}
-\frac{1}{2} \epsilon_{a b} E_{d}^{a b c} \nabla_{c}[\chi, \chi]^{d} & =-\epsilon_{a b} E_{d}^{a b c} \nabla_{c}\left(\chi^{e} \nabla_{e} \chi^{d}\right) \\
& =-\epsilon_{a b} E_{d}^{a b c}\left(\chi^{e} \nabla_{(c} \nabla_{e)} \chi^{d}+\nabla_{c} \chi^{e} \nabla_{e} \chi^{d}\right)+\text { n.d. }
\end{aligned}
$$


which uses identity A.6. When added to (B.4), the second derivative terms cancel since $\chi^{e}$ is an $\mathcal{S}$ one form, so $\left(\nabla_{(c} \nabla_{e)} \chi^{d}\right) \chi^{e}=-\chi^{e} \nabla_{(c} \nabla_{e)} \chi^{d}$. This shows that (B.3) does not depend on second derivatives of $\chi^{d}$.

Open Access. This article is distributed under the terms of the Creative Commons Attribution License (CC-BY 4.0), which permits any use, distribution and reproduction in any medium, provided the original author(s) and source are credited.

\section{References}

[1] H. Casini, M. Huerta and J.A. Rosabal, Remarks on entanglement entropy for gauge fields, Phys. Rev. D 89 (2014) 085012 [arXiv:1312.1183] [inSPIRE].

[2] S. Ryu and T. Takayanagi, Holographic derivation of entanglement entropy from AdS/CFT, Phys. Rev. Lett. 96 (2006) 181602 [hep-th/0603001] [INSPIRE].

[3] T. Faulkner, A. Lewkowycz and J. Maldacena, Quantum corrections to holographic entanglement entropy, JHEP 11 (2013) 074 [arXiv:1307.2892] [INSPIRE].

[4] X. Dong, D. Harlow and A.C. Wall, Reconstruction of bulk operators within the entanglement wedge in gauge-gravity duality, Phys. Rev. Lett. 117 (2016) 021601 [arXiv:1601.05416] [INSPIRE].

[5] D.L. Jafferis, A. Lewkowycz, J. Maldacena and S.J. Suh, Relative entropy equals bulk relative entropy, JHEP 06 (2016) 004 [arXiv:1512.06431] [INSPIRE].

[6] B. Czech, J.L. Karczmarek, F. Nogueira and M. Van Raamsdonk, The gravity dual of a density matrix, Class. Quant. Grav. 29 (2012) 155009 [arXiv:1204.1330] [INSPIRE].

[7] J. Cotler, P. Hayden, G. Salton, B. Swingle and M. Walter, Entanglement wedge reconstruction via universal recovery channels, arXiv:1704.05839 [INSPIRE].

[8] D. Harlow, The Ryu-Takayanagi formula from quantum error correction, Commun. Math. Phys. 354 (2017) 865 [arXiv: 1607.03901] [INSPIRE].

[9] A. Almheiri, X. Dong and D. Harlow, Bulk locality and quantum error correction in AdS/CFT, JHEP 04 (2015) 163 [arXiv: 1411.7041] [INSPIRE].

[10] D.D. Blanco, H. Casini, L.-Y. Hung and R.C. Myers, Relative entropy and holography, JHEP 08 (2013) 060 [arXiv: 1305.3182] [INSPIRE].

[11] J. Bhattacharya, M. Nozaki, T. Takayanagi and T. Ugajin, Thermodynamical property of entanglement entropy for excited states, Phys. Rev. Lett. 110 (2013) 091602 [arXiv: 1212.1164] [INSPIRE].

[12] N. Lashkari, M.B. McDermott and M. Van Raamsdonk, Gravitational dynamics from entanglement 'thermodynamics', JHEP 04 (2014) 195 [arXiv: 1308.3716] [INSPIRE].

[13] T. Faulkner, M. Guica, T. Hartman, R.C. Myers and M. Van Raamsdonk, Gravitation from entanglement in holographic CFTs, JHEP 03 (2014) 051 [arXiv: 1312.7856] [INSPIRE].

[14] B. Swingle and M. Van Raamsdonk, Universality of gravity from entanglement, arXiv: 1405.2933 [INSPIRE].

[15] T. Faulkner, Bulk emergence and the RG flow of entanglement entropy, JHEP 05 (2015) 033 [arXiv: 1412.5648] [INSPIRE]. 
[16] T. Faulkner et al., Nonlinear gravity from entanglement in conformal field theories, JHEP 08 (2017) 057 [arXiv : 1705.03026] [INSPIRE].

[17] T. Jacobson, Entanglement equilibrium and the Einstein equation, Phys. Rev. Lett. 116 (2016) 201101 [arXiv: 1505.04753] [INSPIRE].

[18] H. Casini, D.A. Galante and R.C. Myers, Comments on Jacobson's "Entanglement equilibrium and the Einstein equation", JHEP 03 (2016) 194 [arXiv:1601.00528] [INSPIRE].

[19] A.J. Speranza, Entanglement entropy of excited states in conformal perturbation theory and the Einstein equation, JHEP 04 (2016) 105 [arXiv: 1602.01380] [INSPIRE].

[20] P. Bueno, V.S. Min, A.J. Speranza and M.R. Visser, Entanglement equilibrium for higher order gravity, Phys. Rev. D 95 (2017) 046003 [arXiv:1612.04374] [INSPIRE].

[21] M. Van Raamsdonk, Building up spacetime with quantum entanglement, Gen. Rel. Grav. 42 (2010) 2323 [arXiv: 1005. 3035] [INSPIRE].

[22] R.D. Sorkin, On the entropy of the vacuum outside a horizon, in Tenth International Conference on General Relativity and Gravitation, Contributed Papers volume II, B. Bertotti et al. eds., Consiglio Nazionale Delle Ricerche, Roma, Italy (1983), arXiv:1402.3589 [INSPIRE].

[23] L. Bombelli, R.K. Koul, J. Lee and R.D. Sorkin, A quantum source of entropy for black holes, Phys. Rev. D 34 (1986) 373 [INSPIRE].

[24] M. Srednicki, Entropy and area, Phys. Rev. Lett. 71 (1993) 666 [hep-th/9303048] [INSPIRE].

[25] V.P. Frolov and I. Novikov, Dynamical origin of the entropy of a black hole, Phys. Rev. D 48 (1993) 4545 [gr-qc/9309001] [INSPIRE].

[26] L. Susskind and J. Uglum, Black hole entropy in canonical quantum gravity and superstring theory, Phys. Rev. D 50 (1994) 2700 [hep-th/9401070] [INSPIRE].

[27] T. Jacobson, Black hole entropy and induced gravity, gr-qc/9404039 [INSPIRE].

[28] J.H. Cooperman and M.A. Luty, Renormalization of entanglement entropy and the gravitational effective action, JHEP 12 (2014) 045 [arXiv: 1302.1878] [INSPIRE].

[29] C.G. Callan Jr. and F. Wilczek, On geometric entropy, Phys. Lett. B 333 (1994) 55 [hep-th/9401072] [INSPIRE].

[30] D.N. Kabat, Black hole entropy and entropy of entanglement, Nucl. Phys. B 453 (1995) 281 [hep-th/9503016] [INSPIRE].

[31] D.V. Fursaev and G. Miele, Cones, spins and heat kernels, Nucl. Phys. B 484 (1997) 697 [hep-th/9605153] [INSPIRE].

[32] S.N. Solodukhin, Newton constant, contact terms and entropy, Phys. Rev. D 91 (2015) 084028 [arXiv: 1502.03758] [INSPIRE].

[33] W. Donnelly and A.C. Wall, Do gauge fields really contribute negatively to black hole entropy?, Phys. Rev. D 86 (2012) 064042 [arXiv:1206.5831] [INSPIRE].

[34] W. Donnelly and A.C. Wall, Entanglement entropy of electromagnetic edge modes, Phys. Rev. Lett. 114 (2015) 111603 [arXiv:1412.1895] [INSPIRE].

[35] W. Donnelly and A.C. Wall, Geometric entropy and edge modes of the electromagnetic field, Phys. Rev. D 94 (2016) 104053 [arXiv: 1506.05792] [INSPIRE]. 
[36] W. Donnelly and L. Freidel, Local subsystems in gauge theory and gravity, JHEP 09 (2016) 102 [arXiv: 1601.04744$]$ [INSPIRE].

[37] E. Witten, Interacting Field Theory of Open Superstrings, Nucl. Phys. B 276 (1986) 291 [INSPIRE].

[38] C. Crnkovic and E. Witten, Covariant description of canonical formalism in geometrical theories, in Three hundred years of gravitation, S.W. Hawking and W. Israel eds., Cambridge University Press, Cambridge U.K. (1987).

[39] C. Crnkovic, Symplectic geometry of the covariant phase space, superstrings and superspace, Class. Quant. Grav. 5 (1988) 1557 [INSPIRE].

[40] A. Ashtekar, L. Bombelli and O. Reula, The covariant phase space of asymptotically flat gravitational fields, in Mechanics, analysis and geometry: 200 years after Lagrange, M. Francaviglia ed., Elsevier Science Publishers, Amsterdam The Hetherlands (1991).

[41] S. Carlip, The statistical mechanics of the $(2+1)$-dimensional black hole, Phys. Rev. D 51 (1995) 632 [gr-qc/9409052] [INSPIRE].

[42] S. Carlip, Statistical mechanics and black hole thermodynamics, Nucl. Phys. Proc. Suppl. 57 (1997) 8 [gr-qc/9702017] [inSPIRE].

[43] T. Jacobson, G. Kang and R.C. Myers, On black hole entropy, Phys. Rev. D 49 (1994) 6587 [gr-qc/9312023] [INSPIRE].

[44] J.D. Brown and M. Henneaux, Central charges in the canonical realization of asymptotic symmetries: an example from three-dimensional gravity, Commun. Math. Phys. 104 (1986) 207 [INSPIRE].

[45] S. Carlip, Effective conformal descriptions of black hole entropy, Entropy 13 (2011) 1355 [arXiv: 1107.2678] [INSPIRE].

[46] R.M. Wald, Black hole entropy is the Noether charge, Phys. Rev. D 48 (1993) R3427 [gr-qc/9307038] [INSPIRE].

[47] V. Iyer and R.M. Wald, Some properties of Noether charge and a proposal for dynamical black hole entropy, Phys. Rev. D 50 (1994) 846 [gr-qc/9403028] [InSPIRE].

[48] M. Geiller, Edge modes and corner ambiguities in 3d Chern-Simons theory and gravity, Nucl. Phys. B 924 (2017) 312 [arXiv: 1703.04748] [INSPIRE].

[49] G. Compere, Symmetries and conservation laws in Lagrangian gauge theories with applications to the mechanics of black holes and to gravity in three dimensions, arXiv:0708.3153 [INSPIRE].

[50] J. Lee and R.M. Wald, Local symmetries and constraints, J. Math. Phys. 31 (1990) 725 [INSPIRE].

[51] S. Lang, Differential manifolds, Springer, Germany (1985).

[52] R.M. Wald, General relativity, University of Chicago Press, Chicago, U.S.A. (1984).

[53] S.B. Giddings, D. Marolf and J.B. Hartle, Observables in effective gravity, Phys. Rev. D 74 (2006) 064018 [hep-th/0512200].

[54] T. Andrade, W.R. Kelly and D. Marolf, Einstein-Maxwell Dirichlet walls, negative kinetic energies and the adiabatic approximation for extreme black holes, Class. Quant. Grav. 32 (2015) 195017 [arXiv: 1503.03915] [INSPIRE]. 
[55] T. Andrade, W.R. Kelly, D. Marolf and J.E. Santos, On the stability of gravity with Dirichlet walls, Class. Quant. Grav. 32 (2015) 235006 [arXiv:1504.07580] [InSPIRE].

[56] T. Andrade and D. Marolf, Asymptotic Symmetries from finite boxes, Class. Quant. Grav. 33 (2016) 015013 [arXiv: 1508.02515] [INSPIRE].

[57] V. Iyer and R.M. Wald, A comparison of Noether charge and Euclidean methods for computing the entropy of stationary black holes, Phys. Rev. D 52 (1995) 4430 [gr-qc/9503052] [INSPIRE].

[58] D.G.B. Edelen, Applied exterior calculus, Dover Publications, U.S.A. (2005).

[59] X. Dong, Holographic entanglement entropy for general higher derivative gravity, JHEP 01 (2014) 044 [arXiv: 1310.5713] [INSPIRE].

[60] J. Camps, Generalized entropy and higher derivative Gravity, JHEP 03 (2014) 070 [arXiv: 1310.6659] [INSPIRE].

[61] R.-X. Miao and W.-z. Guo, Holographic entanglement entropy for the most general higher derivative gravity, JHEP 08 (2015) 031 [arXiv:1411.5579] [INSPIRE].

[62] A.C. Wall, A second law for higher curvature gravity, Int. J. Mod. Phys. D 24 (2015) 1544014 [arXiv: 1504.08040] [INSPIRE].

[63] R.M. Wald, On identically closed forms locally constructed from a field, J. Math. Phys. 31 (1990) 2378.

[64] S. Carlip, Entropy from conformal field theory at Killing horizons, Class. Quant. Grav. 16 (1999) 3327 [gr-qc/9906126] [INSPIRE].

[65] S. Silva, Black hole entropy and thermodynamics from symmetries, Class. Quant. Grav. 19 (2002) 3947 [hep-th/0204179] [INSPIRE].

[66] G. Barnich and F. Brandt, Covariant theory of asymptotic symmetries, conservation laws and central charges, Nucl. Phys. B 633 (2002) 3 [hep-th/0111246] [INSPIRE].

[67] L. Freidel, A. Perez and D. Pranzetti, Loop gravity string, Phys. Rev. D 95 (2017) 106002 [arXiv: 1611.03668] [INSPIRE].

[68] G.W. Gibbons and S.W. Hawking, Action Integrals and Partition Functions in Quantum Gravity, Phys. Rev. D 15 (1977) 2752 [InSPIRE].

[69] V.E. Hubeny, M. Rangamani and T. Takayanagi, A Covariant holographic entanglement entropy proposal, JHEP 07 (2007) 062 [arXiv:0705.0016] [INSPIRE].

[70] X. Dong and A. Lewkowycz, Entropy, extremality, euclidean variations and the equations of motion, JHEP 01 (2018) 081 [arXiv: 1705.08453] [INSPIRE].

[71] R.-X. Miao, Universal terms of entanglement entropy for 6d CFTs, JHEP 10 (2015) 049 [arXiv: 1503.05538] [INSPIRE].

[72] J. Camps, Gravity duals of boundary cones, JHEP 09 (2016) 139 [arXiv:1605.08588] [INSPIRE].

[73] W. Donnelly, Decomposition of entanglement entropy in lattice gauge theory, Phys. Rev. D 85 (2012) 085004 [arXiv:1109.0036] [INSPIRE].

[74] W. Donnelly, Entanglement entropy and nonabelian gauge symmetry, Class. Quant. Grav. 31 (2014) 214003 [arXiv:1406.7304] [InSPIRE].

[75] J. Lin, Ryu-Takayanagi area as an entanglement edge term, arXiv:1704.07763 [INSPIRE]. 
[76] H.W. Hamber, Quantum gravity on the lattice, Gen. Rel. Grav. 41 (2009) 817 [arXiv: 0901.0964] [INSPIRE].

[77] T. Jacobson and A. Speranza, to appear.

[78] B. Dittrich, P.A. Hoehn, T.A. Koslowski and M.I. Nelson, Chaos, Dirac observables and constraint quantization, arXiv:1508.01947 [INSPIRE].

[79] S. Hollands and R.M. Wald, Stability of black holes and black branes, Commun. Math. Phys. 321 (2013) 629 [arXiv:1201.0463] [INSPIRE].

[80] N. Lashkari and M. Van Raamsdonk, Canonical energy is quantum Fisher information, JHEP 04 (2016) 153 [arXiv: 1508.00897] [INSPIRE].

[81] M.J.S. Beach, J. Lee, C. Rabideau and M. Van Raamsdonk, Entanglement entropy from one-point functions in holographic states, JHEP 06 (2016) 085 [arXiv:1604.05308] [INSPIRE].

[82] T. Jacobson, J.M.M. Senovilla and A. Speranza, Areas of geodesic balls and the Bel-Robinson tensor, arXiv:1710.07379.

[83] L.B. Szabados, Quasi-local energy-momentum and angular momentum in general relativity, Living Rev. Relativ. 12 (2009) 4.

[84] J.M.M. Senovilla, Superenergy tensors, Class. Quant. Grav. 17 (2000) 2799 [gr-qc/9906087] [INSPIRE].

[85] T. Jacobson and A. Mohd, Black hole entropy and Lorentz-diffeomorphism Noether charge, Phys. Rev. D 92 (2015) 124010 [arXiv:1507.01054] [INSPIRE].

[86] K. Prabhu, The first law of black hole mechanics for fields with internal gauge freedom, Class. Quant. Grav. 34 (2017) 035011 [arXiv:1511.00388] [INSPIRE].

[87] I. Kolář, P.W. Michor and J. Slovák, Natural operators in differential geometry, Springer, Germany (1993). 